\title{
A Novel Discriminant Approximation of Periodic Differential Equations
}

\author{
C.A. Franco ${ }^{\mathrm{a}, 1, *}$, J. Collado ${ }^{\mathrm{a}}$ \\ ${ }^{a}$ Centro de Investigacion y de Estudios Avanzados del Instituto Politecnico Nacional, Mexico City, Mexico. Instituto \\ Politecnico Nacional Av. No 2508, 07360
}

\begin{abstract}
A new approximation of the discriminant of a second order periodic differential equation is presented as a recursive summation of the evaluation of its excitation function at different values of time. The new approximation is obtained, at first, by means of Walsh functions and then, by using some algebraic properties the dependence on the Walsh functions is eliminated. This new approximation is then used to calculate the boundaries of stability. We prove that by letting the summation elements number approach to infinite, the discriminant approximation can be rewritten as a summation of definite integrals. Finally we prove that the definite integrals summation is equivalent to the discriminant approximation made by Lyapunov which consists in an alternating series of coefficients defined by multiple definite integrals, that is, a series of the form $A=A_{0}-A_{1}+\ldots+(-1)^{n} A_{n}$, where each coefficient $A_{n}$ is defined as an $n$-multiple definite integral.
\end{abstract}

Keywords: Hill equation, Discriminant approximation, Walsh functions

\section{Notation}

$\begin{array}{ll}\ddot{x}+(\alpha+\beta p(t)) x= & 0 \\ t & \text { Independent Variable } \\ p(t) & =p(t+\tau), \text { Excitation Function } \\ \tau & \text { Differential Equation Period } \\ \phi_{i} & \text { Characteristic Multipliers } \\ \Delta(\alpha, \beta) \text { or } \Delta(\lambda) & \text { Discriminant } \\ A & \text { Lyapunov Characteristic Constant } \\ \Phi\left(t, t_{0}\right) & \text { State Transition Matrix } \\ M & \text { Monodromy Matrix }(\Phi(\tau, 0)) \\ \lambda_{i}, \lambda_{i}^{\prime} & |\Delta(\lambda)|=2,|\Delta(\lambda)|=-2 \\ w_{n}(t) & n-t h \text { Walsh Function } \\ \bar{w}_{n}(t) & \text { Vector of Walsh Functions } \in \mathbb{R}^{n \times 1} \\ W_{H} & \text { Walsh Matrix } \\ P & \text { Walsh Function Integration Operator } \\ \nu_{n}, r_{n}, \ldots & \text { Real Constants } \\ \bar{\nu}, \bar{r}, \ldots & \text { Real Constant Vectors } \\ I_{n} & \text { Identity Matrix } \\ \Lambda_{i}^{(m)} & \text { Permutation Matrix } \\ p_{n} & =p\left(n \frac{\tau}{2^{k}}\right) \\ e_{1} & =[1 \quad 0 \quad \ldots \\ y_{1} & \end{array}$

$\begin{array}{ll}\text { Hill Equation } & =\left[\begin{array}{llll}0 & 0 & \ldots & 1\end{array}\right]^{T} \\ e_{2^{k}} & =\left[\begin{array}{llll}1 & 1 & \ldots & 1\end{array}\right]^{T} \\ e & p(t) \text { Sampling Matrix } \\ \Lambda_{\gamma} & \text { Discriminant Sampling Matrices } \\ \Gamma, \Gamma_{P} & =W_{H} \Lambda_{\bar{r}} W_{H} \\ \bar{\Lambda}_{\bar{r}} & =W_{H} P W_{H} \\ \bar{P} & =W_{H}\left(P^{-1}\right) W_{H} \\ \bar{P}^{-1} & =W_{H}^{-1} W_{H} \\ \bar{\Gamma} & =W_{H}^{-1} \Gamma_{P} W_{H} \\ \bar{\Gamma}_{P} & 2^{k}-n \text { entry of the last column of } \bar{\Gamma}^{k} \\ b_{n} & =2_{i=0}^{h} b_{i} \\ c_{n} & =\sum_{i=0}^{h} c_{i} \\ S_{h} & \left.=\frac{2^{2 k}}{2^{2 k+2}+\tau^{2}\left(\alpha+\beta p_{2}{ }^{2} h\right.}\right) \\ Z_{h} & =\alpha+\beta p_{2^{k}-h} \\ \psi_{h} & =\alpha+\frac{\beta}{2}\left(p_{2^{k}-h}+p_{2^{k}-h+1}\right) \\ \xi_{h} & =\frac{\tau}{2^{k}} \\ \mu_{h} & =\alpha+\beta p(t) \\ \delta & \end{array}$

\section{Introduction}

$$
=\left[\begin{array}{cccc}
1 & 0 & \ldots & 0
\end{array}\right]^{T}
$$

Linear periodic differential equations can describe the dynamical behaviour of a large number of mechanical systems. They arise quite frequently as the result of linearising a non-linear system about a periodic

\footnotetext{
* Corresponding author

Email addresses: cfranco@ctrl.cinvestav.mx (C.A. Franco), jcollado@ctrl.cinvestav.mx (J. Collado)

${ }^{1}$ The first author, acknowledges the financial support of CONACyt and CINVESTAV.
} 
solution. An important second order example is the Hill equation

$$
\ddot{x}+(\alpha+\beta p(t)) x=0, p(t+\tau)=p(t)
$$

where $\tau$ is the minimum period of the excitation function $p(t)$, and $p(t)$ is assumed piecewise continuous. Equation (1.1) has been used to describe problems in engineering and physics, including problems in mechanics, astronomy and the theory of electric circuits [1, 2]. As is well known the analytic solution of (1.1) can not been obtained and the analysis of stability hinge on the numerical calculation of its solutions.

We can prove that the stability of the solutions of the periodic differential equation (1.1) is defined by an autonomous function which depends only on its constant parameters $\alpha$ and $\beta$, and on the minimum period of its excitation function $p(t)$. This remarkable function is known as the discriminant associated to the Hill equation (1.1) and it is denoted as $\Delta(\alpha, \beta)$. In [3] Lyapunov obtained an approximation of the discriminant in terms of the alternating serie:2

$$
A=A_{0}-A_{1}+A_{2}-A_{3}+\ldots+(-1)^{n} A_{n}+\ldots
$$

the coefficients $A_{k}$ are defined, applying successive approximation [4], as

$$
\begin{aligned}
A_{0}= & 1 \\
A_{1}= & \frac{\tau}{2} \int_{0}^{\tau} q(t) d t \\
A_{2}= & \frac{1}{2} \int_{0}^{\tau} \int_{0}^{t_{1}}\left(\tau-t_{1}+t_{2}\right)\left(t_{1}-t_{2}\right) q\left(t_{1}\right) q\left(t_{2}\right) d t_{2} d t_{1} \\
& \vdots \\
A_{n}= & \frac{1}{2} \int_{0}^{\tau} \int_{0}^{t_{1}} \ldots \int_{0}^{t_{n-1}}\left(\tau-t_{1}+t_{n}\right)\left(t_{1}-t_{2}\right) \ldots\left(t_{n-1}-t_{n}\right) q\left(t_{1}\right) \ldots q\left(t_{n}\right) d t_{1} d t_{2} \ldots d t_{n}
\end{aligned}
$$

where $q(t)=\alpha+\beta p(t)$ and $\tau$ is the minimum period of $p(t)$, i.e. $q(t+\tau)=q(t)$. In $[5]$ Lyapunov did a detailed analysis of his approximation. He obtained the series (1.2) by considering the equation

$$
\ddot{x}+\lambda q(t) x=0
$$

instead of (1.1), and then expanding the characteristic constant $A(\lambda)$ in powers of $\lambda$, i.e. $A(\lambda)=A_{0}-$ $\lambda A_{1}+\lambda^{2} A_{2} \ldots+(-\lambda)^{n} A_{n}+\ldots$, and then setting $\lambda=1$ for obtaining (1.2). Some other discriminant approximation may be found in [6] and in [7], the former approximation is based on some properties of the Sturm-Liouville problem and the latter is based on the successive approximation method.

For clarifying the notion of discriminant $\Delta(\alpha, \beta)$, let $x_{1}$ and $x_{2}$ be two solutions of (1.1) subject to the initial conditions

$$
\begin{aligned}
& x_{1}(0)=1, x_{2}(0)=0 \\
& \dot{x}_{1}(0)=0, \dot{x}_{2}(0)=1
\end{aligned}
$$

It is known [8] that if $\rho$ is solution of the characteristic equation

$$
\rho^{2}-\left(x_{1}(\tau)+\dot{x}_{2}(\tau)\right) \rho+1=0
$$

then (1.1) has at least one solution of the form

$$
x(\tau+t)=\rho x(t)
$$

\footnotetext{
${ }^{2}$ The discriminant was denoted by Lyapunov as the letter $A$. Most of the literature uses the $\Delta(\alpha, \beta)$ notation. The main difference between both of them is that the former is one half of the latter, that is $A=\frac{1}{2} \Delta(\alpha, \beta)$.
} 
the constant $\Delta(\alpha, \beta)=x_{1}(\tau)+\dot{x}_{2}(\tau)$ is known as the discriminant of (1.1).

The roots of the characteristic equation are

$$
\rho_{1,2}=\frac{\Delta(\alpha, \beta) \pm \sqrt{\Delta(\alpha, \beta)^{2}-4}}{2}
$$

from where one can notice that: If $-2<\Delta(\alpha, \beta)<2$, the roots $\rho_{1,2}$ are complex conjugated numbers and lie on the unitary circle, thus $x_{1}(t)$ and $x_{2}(t)$ are bounded so all the solutions of (1.1) are bounded: If $\Delta(\alpha, \beta)= \pm 2, \rho= \pm 1$, there is at least one $\tau$-periodic or $\tau$-anti periodic solution 3 and if the modulo of $\Delta(\alpha, \beta)$ is greater than 2 then, there is one bounded and one unstable solution. So the stability of the solutions of (1.1) are determined by its discriminant $\Delta(\alpha, \beta)$, for further information see section 2 .

The aim of the present work is: a) to introduce a new approximation for the discriminant $\Delta(\alpha, \beta)$. This is accomplished by doing a series of assumptions on a new approximation obtained by means of Wash series and then reducing it to a recursive summation, in terms of evaluation of the excitation function at different values of time $t_{n} \in[0, \tau], n=1,2, \ldots$; and, b) based on the new form of $\Delta(\alpha, \beta)$, we give an alternative proof to the discriminant approximation obtained by Lyapunov in [3] and [5]. The new approximation may be seen as a "discrete" form of the Lyapunov approximation.

This work is structured as follows: In section 2 we give a brief introduction to the theory of periodic differential equations and Walsh series; Section 3 and 4 are dedicated to give a rough approximation of the discriminant of (1.1); in section 5 we give the new approximation of $\Delta(\alpha, \beta)$ and the alternative proof of the Lyapunov approximation is done; and in section 6 we obtain the transition curves of a Hill equation.

\section{Preliminaries}

\subsection{Background for 2nd order linear periodic differential equations}

Consider the linear periodic equation $\ddot{x}+(\alpha+\beta p(t)) x=0$ where $p(\tau+t)=p(t)$, by the usual change of variable $z_{1}=x$ and $z_{2}=\dot{x}$ the differential equation can be rewritten as

$$
\left[\begin{array}{c}
\dot{z}_{1} \\
\dot{z}_{2}
\end{array}\right]=\left[\begin{array}{cc}
0 & 1 \\
-(\alpha+\beta p(t)) & 0
\end{array}\right]\left[\begin{array}{l}
z_{1} \\
z_{2}
\end{array}\right]
$$

It is well known that the general solution of (2.1) is the state transition matrix $\Phi\left(t, t_{0}\right)$ whose columns are linearly independent solutions of (2.1) subject to the initial conditions $\Phi\left(t_{0}, t_{0}\right)=I_{2}$, in other words, let $x_{1}$ and $x_{2}$ be two linearly solutions of the periodic equation $\ddot{x}+(\alpha+\beta p(t)) x=0$ subject to the initial conditions

$$
\begin{array}{ll}
x_{1}\left(t_{0}\right)=1 & x_{2}\left(t_{0}\right)=0 \\
\dot{x}_{1}\left(t_{0}\right)=0 & \dot{x}_{2}\left(t_{0}\right)=1
\end{array}
$$

then the state transition matrix is

$$
\Phi\left(t, t_{0}\right)=\left[\begin{array}{cc}
x_{1}(t) & x_{2}(t) \\
\dot{x}_{1}(t) & \dot{x}_{2}(t)
\end{array}\right], \quad t \in(-\infty, \infty)
$$

The importance of the matrix $\Phi\left(t, t_{0}\right)$ lies in the fact that all solutions of any differential equation, in particular our equation (2.1), can be expressed in terms of the transition matrix as $z(t)=\Phi\left(t, t_{0}\right) z\left(t_{0}\right)$, for all $z\left(t_{0}\right)$.

The matrix $\Phi\left(t, t_{0}\right)$ of a periodic system may be written as a multiplication of three matrices, two of them are time dependent matrices and the other is a constant matrix; it can be proved that one of the time dependent matrices is bounded and periodic, and the other is an exponential one: the former gives us information about the phase of the solutions; and the latter contains information about the growth of the solutions, see [9, 10]. The next Theorem, due to Floquet, gives us the mentioned factorization

\footnotetext{
${ }^{3} \mathrm{~A}$ solution $x(t)$ is $\tau$-anti periodic if $x(t+\tau)=-x(t)$.
} 
Theorem 2.1. The state transition matrix $\Phi\left(t, t_{0}\right) \in \mathbb{R}^{2 \times 2}$ associated to the periodic differential equation (2.1) has the form

$$
\Phi\left(t, t_{0}\right)=P^{-1}(t) e^{B\left(t-t_{0}\right)} P\left(t_{0}\right)
$$

where $P(t)$ and $B$ are $2 \times 2$ matrices, $P(t+\tau)=P(t)$ and $B$ is a constant matrix, not necessarily real.

Proof. The proof can be found in [1]].

If we set $t_{0}=0$ then the factorization made in Theorem 2.1 is reduced to $\Phi(t, 0)=P^{-1}(t) e^{B t}$, this follows from the fact that $P^{-1}(t)=\Phi(t, 0) e^{-B t}$ so if $t=0$ then, $P(0)=I_{2}$.

Theorem 2.1 implies the following: for $t>0$, set $t=k \tau+t_{1}, t_{1} \in[0, \tau)$ where $k$ is a non-negative integer and $t_{0}=0$, by the property $\Phi\left(t_{2}, t_{0}\right)=\Phi\left(t_{2}, t_{1}\right) \Phi\left(t_{1}, t_{0}\right)$ we can write any solution of (2.1) as

$$
\begin{aligned}
z(t) & =\Phi(t, 0) z(0) \\
& =\Phi\left(k \tau+t_{1}, 0\right) z(0) \\
& =\Phi\left(k \tau+t_{1},(k-1) \tau\right) \Phi((k-1) \tau,(k-2) \tau) \ldots \Phi(\tau, 0) z(0)
\end{aligned}
$$

defining a matrix $M=\Phi(\tau, 0)$, the solution $z(t)$ is

$$
z(t)=\Phi\left(t_{1}, 0\right) M^{k} z(0)
$$

then, the stability of any solution $z(t)$ depends on the matrix $M$, i.e. since $\Phi\left(t_{1}, 0\right)$ for $t_{1} \in[0, \tau)$ and $z(0)$ are bounded and using the well-known fact that: if $\sigma(M)=\left\{\rho_{1}, \rho_{2}, \ldots, \rho_{n}\right\}$, then $\sigma\left(M^{k}\right)=\left\{\rho_{1}^{k}, \rho_{2}^{k}, \ldots, \rho_{n}^{k}\right\}$, the only factor of the solution that could grow without bound, as time $t$ increases, is the matrix $M^{k}$, the matrix $M$ is known as the monodromy matrix associated to (2.1). In fact, from equation (2.2) we can notice that one can obtain any solution of (2.1) by only knowing the state transition matrix at the interval $t \in[0, \tau]$. And then, we can state the following

Lemma 2.2. Let $\rho_{i}$ be the eigenvalues of the monodromy matrix $M$ then the solutions of (2.1) are

- Asymptotically stable if and only if all $\left|\rho_{i}\right|<1$

- Stable if and only if all $\left|\rho_{i}\right| \leq 1$, and if any $\rho_{i}$ has modulo one, it must be a simple root of the minimal polynomial of $M$.

- Unstable if and only if there is a $\rho_{i}$ such that $\left|\rho_{i}\right|>1$ or if all $\left|\rho_{i}\right| \leq 1$ but one $\rho_{j}:\left|\rho_{j}\right|=1$ and $\rho_{j}$ is a multiple root of the minimal polynomial of $M$.

Remark 2.3. Notice that the system (2.1) can be written as a Hamiltonian system

$$
\left[\begin{array}{l}
\dot{z}_{1} \\
\dot{z}_{2}
\end{array}\right]=\left[\begin{array}{cc}
0 & 1 \\
-1 & 0
\end{array}\right]\left[\begin{array}{cc}
(\alpha+\beta p(t)) & 0 \\
0 & 1
\end{array}\right]\left[\begin{array}{l}
z_{1} \\
z_{2}
\end{array}\right]
$$

thus, its solutions cannot be asymptotically stable, they can only be bounded or unstable. Moreover, the state transition matrix is a symplectic matrix therefore, its eigenvalues are symmetric with respect to the unitary circle [12]. The condition $\rho_{i}= \pm 1$ implies that there exists at least one periodic solution $\left(\rho_{i}=1\right)$ or anti-periodic solution $\left(\rho_{i}=-1\right)$.

We will expand the last part of the latter remark, but first notice that if $M \in \mathbb{R}^{2 \times 2}$ then its characteristic equation is

$$
\operatorname{det}\left(\rho I_{2}-M\right)=\rho^{2}-\operatorname{Trace}(M) \rho+1
$$

the independent term is equal to 1 because of the Liouville theorem [13], and the eigenvalues are

$$
\rho_{1,2}=\frac{\operatorname{Trace}(M) \pm \sqrt{(\operatorname{Trace}(M))^{2}-4}}{2}
$$


if we define the discriminant of the equation (2.1) as

$$
\Delta(\alpha, \beta)=\operatorname{Trace}(M)
$$

then the eigenvalues and therefore the stability of the solutions depend on the discriminant $\Delta(\alpha, \beta)$.

The discriminant of the Hill equation has been extensively studied, see for example [5, 10, 14] among others. One of the most remarkable theorems on this subject was done by Haupt [8], he proved that, for fixed $\beta$, the functions $\Delta(\alpha, \beta)=2$ and $\Delta(\alpha, \beta)=-2$ have an infinite number of zeros, and there exist intervals where $|\Delta(\alpha, \beta)|$ is less than 2 , some where $|\Delta(\alpha, \beta)|$ is larger than 2 and some values of $\alpha, \beta$ where $|\Delta(\alpha, \beta)|=2$.

Theorem 2.4. Let $\Delta(\alpha, \beta)$ be the discriminant of the periodic differential equation $\ddot{x}+(\alpha+\beta p(t)) x=0$, where $p(t)$ is a real valued function and $p(t+\tau)=p(t)$, the coefficients $\alpha$ and $\beta$ are real numbers and $\beta$ is fixed. There exist two infinite sequences

$$
\lambda_{0}<\lambda_{1} \leq \lambda_{2}<\lambda_{3} \leq \ldots
$$

such that $\Delta\left(\lambda_{i}, \beta\right)=2$. And

$$
\lambda_{1}^{\prime} \leq \lambda_{2}^{\prime}<\lambda_{3}^{\prime} \leq \lambda_{4}^{\prime}<\ldots
$$

such that $\Delta\left(\lambda_{i}^{\prime}, \beta\right)=-2$. These sequences interlace in such a way that

$$
\lambda_{0}<\lambda_{1}^{\prime} \leq \lambda_{2}^{\prime}<\lambda_{1} \leq \lambda_{2}<\lambda_{3}^{\prime} \leq \lambda_{4}^{\prime}<\lambda_{3} \leq \ldots
$$

Whenever $\alpha$ lies in one of the intervals

$$
\left(\lambda_{0}, \lambda_{1}^{\prime}\right),\left(\lambda_{2}^{\prime}, \lambda_{1}\right),\left(\lambda_{2}, \lambda_{3}^{\prime}\right),\left(\lambda_{4}^{\prime}, \lambda_{3}\right), \ldots, \text { then }|\Delta(\alpha, \beta)|<2
$$

if $\alpha$ lies in

$$
\begin{aligned}
& \left(-\infty, \lambda_{0}\right),\left(\lambda_{1}^{\prime}, \lambda_{2}^{\prime}\right),\left(\lambda_{1}, \lambda_{2}\right),\left(\lambda_{3}^{\prime}, \lambda_{4}^{\prime}\right), \ldots \text {, then }|\Delta(\alpha, \beta)|>2 \\
& \text { if } \lambda_{k}=\lambda_{k+1} \text { then }\left.\Delta(\alpha, \beta)\right|_{\alpha=\lambda_{k}}=2 \text { and }\left.\frac{\partial \Delta(\alpha, \beta)}{\partial \alpha}\right|_{\alpha=\lambda_{k}}=0 ; \text { and if } \lambda_{k}^{\prime}=\lambda_{k+1}^{\prime} \text { then }\left.\Delta(\alpha, \beta)\right|_{\alpha=\lambda_{k}^{\prime}}=-2 \text { and } \\
& \left.\frac{\partial \Delta(\alpha, \beta)}{\partial \alpha}\right|_{\alpha=\lambda_{k}^{\prime}}=0 .
\end{aligned}
$$

Proof. The proof can be seen in [14] or [8].

Theorem 2.4 implies that, for a fixed $\beta$, the discriminant $\Delta(\alpha, \beta)$ of equation (2.1) has an infinite number of stable and unstable intervals, and those intervals will be bounded by zeros of $\Delta(\alpha, \beta)=2$ and $\Delta(\alpha, \beta)=-2$. If we eliminate the condition on $\beta$, the boundaries characterized by $\Delta(\alpha, \beta)=2$ and $\Delta(\alpha, \beta)=-2$ will define the so called transition curves in the $\alpha-\beta$ plane, Figure 4 in section 6 shows the transition curves for the equation

$$
\ddot{x}+(\alpha+\beta(\cos (t)+\cos (2 t))) x=0
$$

\subsection{Walsh Series}

In this part the main properties of the Walsh functions are described and some of them are proved.

The Walsh functions $w_{n}(t)$ form an ordered set of rectangular waveforms taking only two amplitude values \pm 1 and they form an orthogonal set of $\mathcal{L}_{2}[0,1]$, the Lebesgue space of square integrable functions on $[0,1]$, see [15], i.e.

$$
\int_{0}^{1} h w_{n}(t) w_{m}(t) d t=\left\{\begin{array}{l}
h \text { if } n=m \\
0 \text { if } n \neq m
\end{array}\right.
$$

Walsh functions are defined over a limited time interval $[0,1]$, but it may be transformed in any other interval $[a, b]$. Two arguments are required for completing the definition, a time period $t$ and an ordering number $n$, this number is related to the number of zero crosses of each Walsh function 4 [15].

\footnotetext{
${ }^{4}$ When $\sin (t)$ and $\cos (t)$ are used in a Fourier series, the index is related to frequency; for Walsh functions, the index is called sequency and the analysis based on Walsh functions is called sequency theory.
} 
Remark 2.5. The number of Walsh functions considered is always a power of 2 , i.e. $\left\{w_{0}, w_{1}, \ldots, w_{2^{k}-1}\right\}$ for some positive integer $k$.

The Walsh functions can be obtained in several different ways such as: Rademacher functions [15]

$$
w_{n}(t)=\operatorname{sign}\left[(\sin 2 \pi t)^{b_{0}} \prod_{k=1}^{m}\left(\cos 2^{k} \pi t\right)^{b_{k}}\right]
$$

where $b_{0} \ldots b_{m}$ are the binary bits of the number $n$ expressed in binary i.e. $n=\left(b_{m} b_{m-1} \ldots b_{0}\right)$. They can also be obtained by Boolean synthesis and from Hadamard matrices, being the latter the most known and used. The first eight Walsh functions are shown in Fig. 1

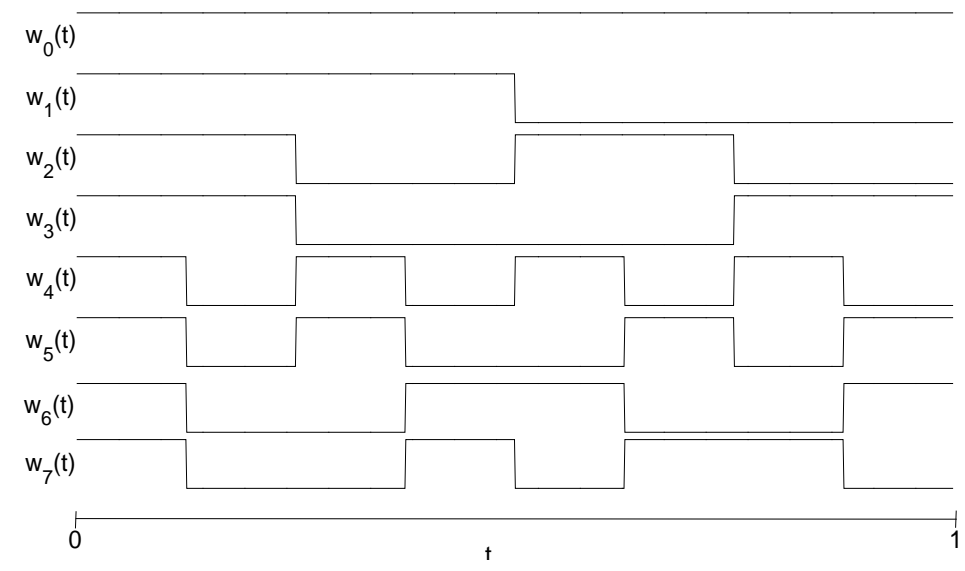

Figure 1: First eight Walsh functions, $w_{0}=1 \forall t \in[0,1]$. Upper values always are +1 and lower values always are -1 .

The representation of Walsh functions by Hadamard matrices is known as Walsh matrix, we will denote it as $W_{H}$, each row corresponds to a Walsh function. The Walsh matrix is obtained by rearranging the rows of a Hadamard matrix 5 . In this work we are rearranging the rows in a dyadic manner [16], which is a different ordering of the same set. The Walsh matrix for the first eight Walsh functions in Fig. 1 is

$$
W_{H}=\left[\begin{array}{cccccccc}
1 & 1 & 1 & 1 & 1 & 1 & 1 & 1 \\
1 & 1 & 1 & 1 & -1 & -1 & -1 & -1 \\
1 & 1 & -1 & -1 & 1 & 1 & -1 & -1 \\
1 & 1 & -1 & -1 & -1 & -1 & 1 & 1 \\
1 & -1 & 1 & -1 & 1 & -1 & 1 & -1 \\
1 & -1 & 1 & -1 & -1 & -1 & -1 & 1 \\
1 & -1 & -1 & 1 & 1 & -1 & -1 & 1 \\
1 & -1 & -1 & 1 & -1 & 1 & 1 & -1
\end{array}\right]
$$

the sign of each row entry correspond to the sign of the Walsh function that is being represented at some time, in other words, the first entry of the first row is $w_{1}(0)$ and the last entry is $w_{1}(1),\left(W_{H}\right)_{1,1}=w_{1}(0)$ and $\left(W_{H}\right)_{1,2^{k}}=w_{1}(1)$. Notice that $W_{H} \in \mathbb{R}^{2^{k} \times 2^{k}}$ is a symmetric and almost orthogonal matrix, $W_{H}^{T}=W_{H}$ and $W_{H}^{-1} W_{H}=2^{k} I_{2^{k}}$, here ${ }^{T}$ is the transpose operator.

\footnotetext{
${ }^{5}$ Recall that Hadamard matrices exist only for dimension $2^{k} \times 2^{k}, k$ being a positive integer. It is consistent with remark 2.5 The entries of such matrices are plus and minus ones, and its rows and columns are mutually orthogonal.
} 
It is known that every function $f(t)$, which is integrable (in the Lebesgue sense), could be represented by a Walsh series [16] defined over the interval $t \in[0,1]$ as

$$
f(t)=\sum_{n=0}^{2^{k}-1} a_{n} w_{n}(t)=\bar{a}^{T} \bar{w}_{2^{k}}(t)
$$

where $\bar{a}^{T}=\left[\begin{array}{lll}a_{0}, & \ldots, & a_{2^{k}-1}\end{array}\right] \in \mathbb{R}^{2^{k}}$ are the weights associated to each Walsh function and $\bar{w}_{2^{k}}(t)=$ $\left[w_{0}(t), \quad \ldots, \quad w_{2^{k}-1}(t)\right]^{T} \in \mathbb{R}^{2^{k}}, k$ is a positive integer number and it is related to the accuracy of the representation (2.4). From now on we will use $2^{k}$ to denote the order of the approximation. The coefficients $a_{n}$ are given by

$$
a_{n}=\int_{0}^{1} f(t) w_{n}(t) d t
$$

equation (2.4) can also be written in terms of the Walsh matrix as $f(t)=\bar{a}^{T} W_{H}$.

A very attractive property of Walsh functions is that the integrals of the function $w_{n}$ which belongs to the finite set $\left\{w_{0}, w_{1}, \ldots, w_{2^{k}-1}\right\}$ can be represented in terms of the set functions [17], in order to illustrate this property, lets take the first integral of $w_{0}(t)$ and then represent it as in (2.4) for $k=2$ and $t \in[0,1]$, so

$$
\int_{0}^{t} w_{0}\left(\tau_{1}\right) d \tau_{1}=t
$$

if one substitutes $f(t)=t$ into (2.5) one obtains

$$
\begin{gathered}
a_{0}=\int_{0}^{1} t w_{0}(t) d t=\frac{1}{2} \quad a_{1}=\int_{0}^{1} t w_{1}(t) d t=-\frac{1}{4} \\
a_{2}=\int_{0}^{1} t w_{2}(t) d t=-\frac{1}{8} \quad a_{3}=\int_{0}^{1} t w_{3}(t) d t=0
\end{gathered}
$$

then the truncated representation (2.4) of the ramp function $f(t)=\int_{0}^{t} w_{0}(t)=t$ is

$$
\int_{0}^{t} w_{0}\left(t_{1}\right) d t_{1}=\left[\begin{array}{llll}
\frac{1}{2} & -\frac{1}{4} & -\frac{1}{8} & 0
\end{array}\right] \bar{w}_{4}(t)
$$

similarly, one can take the first integral of $w_{1}(t), w_{2}(t)$ and $w_{3}(t)$ to obtain

$$
\begin{aligned}
\int_{0}^{t} w_{1}\left(t_{1}\right) d t_{1} & =\left[\begin{array}{llll}
\frac{1}{4} & 0 & 0 & -\frac{1}{8}
\end{array}\right] \bar{w}_{4}(t) \\
\int_{0}^{t} w_{2}\left(t_{1}\right) d t_{1} & =\left[\begin{array}{llll}
\frac{1}{8} & 0 & 0 & 0
\end{array}\right] \bar{w}_{4}(t) \\
\int_{0}^{t} w_{3}\left(t_{1}\right) d t_{1} & =\left[\begin{array}{llll}
0 & \frac{1}{8} & 0 & 0
\end{array}\right] \bar{w}_{4}(t)
\end{aligned}
$$

rewriting the last four equations

$$
\begin{aligned}
\int_{0}^{t} \bar{w}_{4}\left(t_{1}\right) d t_{1} & =\left[\begin{array}{cccc}
\frac{1}{2} & -\frac{1}{4} & -\frac{1}{8} & 0 \\
\frac{1}{4} & 0 & 0 & -\frac{1}{8} \\
\frac{1}{8} & 0 & 0 & 0 \\
0 & \frac{1}{8} & 0 & 0
\end{array}\right] \bar{w}_{4}(t) \\
& =P^{(4)} \bar{w}_{4}(t)
\end{aligned}
$$

with similar arguments one can generalize (2.6) as

$$
\int_{0}^{t} \bar{w}_{m}(t)=P^{(m)} \bar{w}_{m}(t)
$$


where $P^{(m)} \in \mathbb{R}^{m \times m}, m=2^{k}$, and

$$
\begin{aligned}
P^{(n)} & =\left[\begin{array}{cc}
P^{(n-1)} & -\frac{1}{2^{n+1}} I_{2^{n-1}} \\
\frac{1}{2^{n+1}} I_{2^{n-1}} & 0_{2^{n-1}}
\end{array}\right] \\
P^{(0)} & =\frac{1}{2}
\end{aligned}
$$

Matrix $P$ is the Walsh function integration operator. It is worth to notice that $P$ is non-singular since its determinant is $\operatorname{det}(P)=\frac{1}{2^{k}}$.

Another remarkably useful property of Walsh functions is that they are closed under multiplication, the multiplication of Walsh functions is defined as

$$
w_{n}(t) w_{m}(t)=w_{n \oplus m}(t)
$$

where $\oplus$ represents no-carry modulo-2 addition 6 [15]. For example

$$
\begin{aligned}
& w_{0}(t) w_{5}(t)=w_{5}(t) \\
& w_{2}(t) w_{6}(t)=w_{4}(t)
\end{aligned}
$$

It is of great importance to notice that if $w_{n}(t)$ and $w_{m}(t)$ belong to the finite set of Walsh functions

$$
\left\{w_{0}(t), w_{1}(t), \ldots, w_{2^{k}-1}(t)\right\}
$$

then, $w_{n}(t) w_{m}(t)$ may be represented in terms of the finite set of Walsh functions to which they belong. Some other properties will be presented and proved as we need them. In the next section we obtain a numerical approximation of the discriminant of the Hill equation.

\section{Approximation of the discriminant $\Delta(\alpha, \beta)$}

It is well known that the problem of solving the second order differential equation,

$$
\ddot{z}+g(t) z=0, \quad z(0)=a, \dot{z}(0)=b
$$

where $g(t)$ is bounded and for $t \geq 0$, it is equivalent to solving the associated integral equation [18]

$$
z=-\int_{0}^{t} \int_{0}^{t_{1}} g\left(t_{2}\right) x\left(t_{2}\right) d t_{2} d t_{1}+b t+a
$$

There is a vast amount of numerical or pseudo-analytical methods by which one can approximate the solution of (3.1) or (3.2), see for example [19, 20]. In this part we will approximate the solution by means of Walsh functions i.e. we will propose a solution of the form $z=\bar{a}^{T} \bar{w}_{m}$ as in (2.4).

Consider the problem of solving a Hill equation

$$
\ddot{x}+(\alpha+\beta p(t)) x=0 \quad x(0)=x_{0}, \dot{x}(0)=\dot{x}_{0}
$$

where $p(\tau+t)=p(t)$ is a real bounded function, $\alpha, \beta \in \mathbb{R}$ and for $t \in[0, \tau]$. This problem is equivalent to solving the integral equation

$$
x=-\int_{0}^{t} \int_{0}^{t_{1}}\left(\alpha+\beta p\left(t_{2}\right)\right) z\left(t_{2}\right) d t_{2} d t_{1}+\dot{x}_{0} t+x_{0}, \quad 0 \leqslant t_{1} \leqslant t \leqslant \tau
$$

\footnotetext{
${ }^{6}$ For example, the no-carry modulo- 2 addition of 7 and 2 is equal to 5 , since $(111)_{B} \oplus(010)_{B}=(101)_{B}$
} 
We propose a solution of (3.4) in terms of Walsh functions

$$
x=\sum_{n=0}^{2^{k}-1} \nu_{n} w_{n}(t)=\bar{\nu}^{T} \bar{w}_{2^{k}}
$$

here the constant vector $\bar{\nu} \in \mathbb{R}^{2^{k}}$ is unknown and $\bar{w}_{2^{k}}(t)$ is the vector of Walsh functions $\bar{w}_{2^{k}}(t)=$ $\left[\begin{array}{lll}w_{0}(t) & \ldots & w_{2^{k}-1}(t)\end{array}\right]^{T}$. Where $2^{k}$ denotes the order of the approximation and $k$ is an integer positive number. Integrating (3.3) ones we get

$$
\dot{x}=-\alpha \int_{0}^{t} x\left(t_{1}\right) d t_{1}-\beta \int_{0}^{t} p\left(t_{1}\right) x\left(t_{1}\right) d t_{1}+\dot{x}_{0}
$$

expressing the function $p(t)$ in terms of Walsh functions, $p(t)=\sum_{n=0}^{2^{k}-1} r_{n} w_{n}(t)=\bar{r}^{T} \bar{w}_{2^{k}}$, where the real constant vector $\bar{r} \in \mathbb{R}^{2^{k}}$ is known. Substituting (3.5) in the latter equation

$$
\dot{x}=-\alpha \int_{0}^{t} \bar{\nu}^{T} \bar{w}_{2^{k}}\left(t_{1}\right) d t_{1}-\beta \int_{0}^{t} \bar{r}^{T} M_{2^{k}} \bar{\nu} d t_{1}+\dot{x}_{0}, \quad t \in[0, \tau]
$$

where the matrix $M_{2^{k}} \triangleq \bar{w}_{2^{k}} \bar{w}_{2^{k}}^{T}$ has the form

$$
\begin{aligned}
M_{2^{k}}= & {\left[\begin{array}{cccc}
w_{0} w_{0} & w_{1} w_{0} & \cdots & w_{2^{k}-1} w_{0} \\
w_{0} w_{1} & w_{1} w_{1} & \cdots & w_{2^{k}-1} w_{0} \\
\vdots & \vdots & \vdots & \vdots \\
w_{0} w_{2^{k}-1} & w_{1} w_{2^{k}-1} & \cdots & w_{2^{k}-1} w_{2^{k}-1}
\end{array}\right] } \\
= & {\left[\begin{array}{cccc}
w_{0 \oplus 0} & w_{1 \oplus 0} & \cdots & w_{2^{k}-1 \oplus 0} \\
w_{0 \oplus 1} & w_{1 \oplus 1} & \cdots & w_{2^{k}-1 \oplus 1} \\
\vdots & \vdots & \vdots & \vdots \\
w_{0 \oplus 2^{k}-1} & w_{1 \oplus 2^{k}-1} & \cdots & w_{2^{k}-1 \oplus 2^{k}-1}
\end{array}\right] }
\end{aligned}
$$

Notice that the matrix $M_{2^{k}}$ is a symmetric matrix, moreover, the first column of matrix $M_{2^{k}}$ is the vector of Walsh functions $\bar{w}_{2^{k}}(t)$ and the other $2^{k}-1$ columns are permutations of the entries of the first column, that is, the matrix $M_{2^{k}}$ can be written as

$$
M_{2^{k}}(t)=\left[\bar{w}_{2^{k}}(t), \Lambda_{1}^{\left(2^{k}\right)} \bar{w}_{2^{k}}(t), \ldots, \Lambda_{2^{k}-1}^{\left(2^{k}\right)} \bar{w}_{2^{k}}(t)\right]
$$

where each $\Lambda_{i}^{\left(2^{k}\right)}$ is a symmetric permutation matrix, see Lemma in Appendix A.1.

It is desirable to rewrite the second term on the right hand side of (3.6) so the vector $\bar{\nu}^{T}$ pre-multiply and $\bar{w}(t)$ post-multiply some matrix $Q$, i.e. $\bar{r}^{T} M_{2^{k}} \bar{\nu}=\bar{\nu}^{T} Q \bar{w}_{2^{k}}$. For this purpose we introduce the next Lemma, taken from [21]

Lemma 3.1. If $M_{2^{k}}(t)=\bar{w}_{2^{k}} \bar{w}_{2^{k}}^{T}$ and $\gamma \in \mathbb{R}^{2^{k}}$ then $M_{2^{k}} \gamma=\Lambda_{\gamma} \bar{w}_{2^{k}}$ where $\Lambda_{\gamma}=\left[\gamma, \Lambda_{1}^{\left(2^{k}\right)} \gamma, \ldots, \Lambda_{2^{k}-1}^{\left(2^{k}\right)} \gamma\right]$.

Proof. Multiplying the representation (3.7) of $M_{2^{k}}(t)$ by a vector $\gamma \in \mathbb{R}^{2^{k}}$ one obtains

$$
\begin{aligned}
M_{2^{k}}(t) \gamma & =\left[\begin{array}{c}
\bar{w}_{2^{k}}^{T}(t) \gamma \\
\bar{w}_{2^{k}}^{T}(t) \Lambda_{1}^{\left(2^{k}\right)} \gamma \\
\vdots \\
\bar{w}_{2^{k}}^{T}(t) \Lambda_{2^{k}-1}^{\left(2^{k}\right)} \gamma
\end{array}\right]=\left[\begin{array}{c}
\gamma^{T} \bar{w}_{2^{k}}(t) \\
\gamma^{T} \Lambda_{1}^{\left(2^{k}\right)} \bar{w}_{2^{k}}(t) \\
\vdots \\
\gamma^{T} \Lambda_{2^{k}-1}^{\left(2^{k}\right)} \bar{w}_{2^{k}}(t)
\end{array}\right] \\
& =\left[\gamma, \Lambda_{1}^{\left(2^{k}\right)} \gamma, \ldots, \Lambda_{2^{k}-1}^{\left(2^{k}\right)} \gamma\right] \bar{w}_{2^{k}}(t) \\
& =\Lambda_{\gamma} \bar{w}_{2^{k}}(t)
\end{aligned}
$$


since $M_{2^{k}}(t)$ and $\Lambda_{n}^{\left(2^{k}\right)}$ are symmetric matrices.

By latter Lemma and $\int_{0}^{t} \bar{w}_{2^{k}}\left(t_{1}\right) d t_{1}=\tau P \bar{w}_{2^{k}}(t), t \in[0, \tau], P \triangleq P^{\left(2^{k}\right)}$, one can rewrite (3.6) as

$$
\begin{aligned}
\dot{x} & =-\alpha \int_{0}^{t} \bar{\nu}^{T} \bar{w}_{2^{k}}\left(t_{1}\right) d t_{1}-\beta \int_{0}^{t} \bar{\nu}^{T} \Lambda_{\bar{r}} \bar{w}_{2^{k}}\left(t_{1}\right) d t_{1}+\dot{x}_{0} \\
& =-\tau\left(\alpha \bar{\nu}^{T} P+\beta \bar{\nu}^{T} \Lambda_{\bar{r}} P\right) \bar{w}_{2^{k}}(t)+\dot{x}_{0}
\end{aligned}
$$

integrating once again, one obtains

$$
x=-\tau^{2}\left(\alpha \bar{\nu}^{T}+\beta \bar{\nu}^{T} \Lambda_{\bar{r}}\right) P^{2} \bar{w}_{2^{k}}(t)+\tau \dot{x}_{0} e_{1}^{T} P \bar{w}_{2^{k}}(t)+x_{0}
$$

where $e_{1}^{T}=\left[\begin{array}{llll}1 & 0 & \cdots & 0\end{array}\right]$.

From equations (3.5), $x=\bar{\nu}^{T} \bar{w}_{2^{k}}$, and (3.8) one can say

$$
\bar{\nu}^{T} \bar{w}_{2^{k}}(t)=\left(-\tau^{2} \bar{\nu}^{T}\left(\alpha I_{2^{k}}+\beta \Lambda_{\bar{r}}\right) P^{2}+\tau \dot{x}_{0} e_{1}^{T} P+x_{0} e_{1}^{T}\right) \bar{w}_{2^{k}}(t)
$$

so

solving for $\bar{\nu}^{T}$

$$
\bar{\nu}^{T}=-\tau^{2} \bar{\nu}^{T}\left(\alpha I_{2^{k}}+\beta \Lambda_{\bar{r}}\right) P^{2}+\tau \dot{x}_{0} e_{1}^{T} P+x_{0} e_{1}^{T}
$$

where

$$
\bar{\nu}^{T}=\left(\tau \dot{x}_{0} e_{1}^{T} P+x_{0} e_{1}^{T}\right) \Gamma
$$

where

$$
\Gamma=\left(I_{2^{k}}+\tau^{2}\left(\alpha I_{2^{k}}+\beta \Lambda_{\bar{r}}\right) P^{2}\right)^{-1}
$$

notice that $\Gamma \in \mathbb{R}^{2^{k} \times 2^{k}}$ is a large dimensional matrix and it does not have any recognizable pattern.

Then the approximation of the general solution $x$ of the Hill equation (3.3) and its derivative $\dot{x}$, are

$$
\begin{aligned}
x & =\left(\tau \dot{x}_{0} e_{1}^{T} P+x_{0} e_{1}^{T}\right) \Gamma \bar{w}_{2^{k}}(t) \\
\dot{x} & =-\frac{1}{\tau}\left(\tau \dot{x}_{0} e_{1}^{T} P+x_{0} e_{1}^{T}\right)\left(I_{2^{k}}-\Gamma\right) P^{-1} \bar{w}_{2^{k}}(t)+\dot{x}_{0}
\end{aligned}
$$

therefore two linearly independent solutions of (3.4) are

$$
\begin{array}{lll}
x_{1}=e_{1}^{T} \Gamma \bar{w}_{2^{k}}(t), & \text { for } x_{0}=1, & \dot{x}_{0}=0 \\
x_{2}=\tau e_{1}^{T} P \Gamma \bar{w}_{2^{k}}(t), & \text { for } x_{0}=0, & \dot{x}_{0}=1
\end{array}
$$

and the approximation of the state transition matrix $\Phi(t, 0)$ is

$$
\Phi(t, 0)=\left[\begin{array}{cc}
e_{1}^{T} \Gamma \bar{w}_{2^{k}}(t) & \tau e_{1}^{T} P \Gamma \bar{w}_{2^{k}}(t) \\
-\frac{1}{\tau} e_{1}^{T}\left(I_{2^{k}}-\Gamma\right) P^{-1} \bar{w}_{2^{k}}(t) & e_{1}^{T}\left(P \Gamma P^{-1}\right) \bar{w}_{2^{k}}(t)
\end{array}\right], \quad t \in[0, \tau]
$$

Finally the approximation of the discriminant (2.3), $\Delta(\alpha, \beta)=x_{1}(\tau)+\dot{x}_{2}(\tau)$, is

$$
\Delta(\alpha, \beta)=e^{T}\left[\Gamma+P \Gamma P^{-1}\right] \bar{w}_{2^{k}}(\tau)
$$

where $\tau$ is the minimum period of the Hill equation excitation function. The vector $\bar{w}_{2^{k}}(\tau)$ is the last column of the Walsh matrix $W_{H}$. The matrices $\Gamma$ and $P \Gamma P^{-1}$ will be denoted as the discriminant sampling matrices, see remark 4.3 .

Notice that for determining $\Delta(\alpha, \beta)$ we only require the first row of the matrix $\Gamma+P \Gamma P^{-1}$, the main trouble with (3.11) is that we need to obtain the matrix $\Gamma$, in other words, we need to find the inverse matrix of a $2^{k} \times 2^{k}$ matrix where $2^{k}$ is the approximation order and it is related to its accuracy. Nevertheless, (3.11) is easy to treat as we will see in the next section, the non-singularity condition of $\Gamma$ will be treated in section 5 . 


\section{Simplification of the $\Delta(\alpha, \beta)$ approximation}

We had seen that in order to compute $\Delta(\alpha, \beta)$, it is necessary to obtain $\Gamma$ which is the inverse of a large dimensional matrix. In this section we will obtain a simplified version of $\Delta(\alpha, \beta)$, i.e. we will see that the matrix $\Gamma+P \Gamma P^{-1}$ can be rewritten as an upper triangular matrix and the dependence on Walsh functions will be eliminated.

Let $W_{H} \in \mathbb{R}^{2^{k} \times 2^{k}}$ be the representation of the Walsh functions in terms of Hadamard matrix, see section 2 , using the fact that the Walsh functions are orthogonal to each other, one can prove that $W_{H}^{-1}=\frac{1}{2^{k}} W_{H}^{T}=$ $\frac{1}{2^{k}} W_{H}$

We claim that the matrix $\Lambda_{\bar{r}}$ is similar to a diagonal matrix $\bar{\Lambda}_{\bar{r}} \triangleq W_{H}^{-1} \Lambda_{\bar{r}} W_{H}=\operatorname{diag}\left(p_{1}, p_{2}, \ldots, p_{2^{k}}\right)$, where the constants $p_{n}$ are defined as $p_{n}=p\left(n \frac{\tau}{2^{k}}\right), n=1,2, \ldots 2^{k}$, and $p(t)$ is the Hill equation excitation function. In addition, we can say that $p_{n}$ are the eigenvalues of $\Lambda_{\bar{r}}$ and the columns of $W_{H}$ are the eigenvectors associated to each $p_{n}$, namely, $\Lambda_{\bar{r}} W_{H}=W_{H} \operatorname{diag}\left(p_{1}, p_{2}, \ldots, p_{2^{k}}\right)$. This claim follows from Lemma 3.1, for a detailed proof see 22]. Notice that the entries of the diagonal matrix $\bar{\Lambda}_{\bar{r}}$ are the function $p(\cdot)$ evaluated at the end of the $n t h$ interval. The matrix $\bar{\Lambda}_{\bar{r}}$ may be seen as a $p(t)$ sampling matrix.

One can prove that the integration operator $P$ is similar to the upper triangular matrix $\bar{P} \triangleq W_{H} P W_{H}=$ $\frac{1}{2} I_{2^{k}}+Q+Q^{2}+\ldots+Q^{2^{k}-1}$ where $Q$ is the nilpotent matrix

$$
Q=\left[\begin{array}{cccc}
0 & 1 & \cdots & 0 \\
\vdots & \vdots & \ddots & \vdots \\
0 & 0 & \cdots & 1 \\
0 & 0 & \cdots & 0
\end{array}\right] \in \mathbb{R}^{2^{k} \times 2^{k}}
$$

and $\frac{\bar{P}^{-1}}{2^{2 k+2}} \triangleq W_{H} P^{-1} W_{H}=\frac{1}{2} I_{2^{k}}-Q+Q^{2}+\ldots-Q^{2^{k}-1}$, the proof may be found in [23]. For sake of completeness we write the matrices $\bar{\Lambda}_{\bar{r}}, \bar{P}$ and $\bar{P}^{-1}$

$$
\begin{aligned}
& \bar{\Lambda}_{\bar{r}}=\left[\begin{array}{ccccc}
p_{1} & 0 & \cdots & 0 & 0 \\
0 & p_{2} & \cdots & 0 & 0 \\
\vdots & \vdots & \ddots & \vdots & \vdots \\
0 & 0 & \cdots & p_{2^{k}-1} & 0 \\
0 & 0 & \cdots & 0 & p_{2^{k}}
\end{array}\right], \bar{P}=\left[\begin{array}{ccccc}
\frac{1}{2} & 1 & \cdots & 1 & 1 \\
0 & \frac{1}{2} & \cdots & 1 & 1 \\
\vdots & \vdots & \ddots & \vdots & \vdots \\
0 & 0 & \cdots & \frac{1}{2} & 1 \\
0 & 0 & \cdots & 0 & \frac{1}{2}
\end{array}\right] \\
& \text { and } \bar{P}^{-1}=2^{2 k+2}\left[\begin{array}{cccccc}
\frac{1}{2} & -1 & 1 & \cdots & 1 & -1 \\
0 & \frac{1}{2} & -1 & \ddots & -1 & 1 \\
0 & 0 & \frac{1}{2} & \ddots & 1 & -1 \\
\vdots & \vdots & \vdots & \ddots & \vdots & \vdots \\
0 & 0 & 0 & \cdots & \frac{1}{2} & -1 \\
0 & 0 & 0 & \cdots & 0 & \frac{1}{2}
\end{array}\right]
\end{aligned}
$$

Claim 4.1. The matrix $\Gamma=\left(I_{2^{k}}+\tau^{2}\left(\alpha I_{2^{k}}+\beta \Lambda_{R}\right) P^{2}\right)^{-1}$ is almost orthogonal similar to the upper triangular matrix

$$
\bar{\Gamma}=\left(I_{2^{k}}+\frac{\tau^{2}}{2^{2 k}}\left(\alpha I_{2^{k}}+\beta \bar{\Lambda}_{\bar{r}}\right) \bar{P}^{2}\right)^{-1}
$$

Proof. The proof is based on elementary algebraic properties of matrices, one just has to pre-multiply and

\footnotetext{
${ }^{7}$ We say that a square matrix $A$ is almost orthogonal similar to $B$ if $\exists$ an almost orthogonal matrix $R$ such that $B=R A R^{-1}$ and $R^{T} R=\alpha I, \alpha \neq 0$.
} 
post-multiply $\Gamma$ by $W_{H}^{-1}$ and $W_{H}$ respectively and to use the definitions of $\bar{\Lambda}_{\bar{r}}, \bar{P}$ and $\bar{P}^{-1}$

$$
\begin{aligned}
W_{H}^{-1} \Gamma W_{H} & =W_{H}^{-1}\left(I_{2^{k}}+\tau^{2}\left(\alpha I_{2^{k}}+\beta \Lambda_{\bar{r}}\right) P^{2}\right)^{-1} W_{H} \\
& =\left(W_{H}^{-1}\left(I_{2^{k}}+\tau^{2}\left(\alpha I_{2^{k}}+\beta \Lambda_{\bar{r}}\right) P^{2}\right) W_{H}\right)^{-1} \\
& =\left(I_{2^{k}}+\tau^{2} \alpha W_{H}^{-1} P^{2} W_{H}+\tau^{2} \beta W_{H}^{-1} \Lambda_{\bar{r}} P^{2} W_{H}\right)^{-1} \\
& =\left(I_{2^{k}}+\tau^{2} \alpha W_{H}^{-1} P W_{H} W_{H}^{-1} P W_{H}+\tau^{2} \beta W_{H}^{-1} \Lambda_{\bar{r}} W_{H} W_{H}^{-1} P W_{H} W_{H}^{-1} P W_{H}\right)^{-1} \\
& =\left(I_{2^{k}}+\frac{\tau^{2} \alpha}{2^{2 k}} W_{H} P W_{H} W_{H} P W_{H}+\frac{\tau^{2} \beta}{2^{2 k}} W_{H}^{-1} \Lambda_{\bar{r}} W_{H} W_{H} P W_{H} W_{H} P W_{H}\right)^{-1} \\
& =\left(I_{2^{k}}+\frac{\tau^{2} \alpha}{2^{2 k}} \bar{P}^{2}+\frac{\tau^{2} \beta}{2^{2 k}} \bar{\Lambda}_{\bar{r}} \bar{P}^{2}\right)^{-1} \\
& =\left(I_{2^{k}}+\frac{\tau^{2}}{2^{2 k}}\left(\alpha I+\beta \bar{\Lambda}_{\bar{r}}\right) \bar{P}^{2}\right)^{-1} \\
& =\bar{\Gamma}
\end{aligned}
$$

since $\bar{P}$ is an upper triangular matrix; $\bar{P}^{2}$ and $\left(I_{2^{k}}+\frac{\tau^{2}}{2^{2 k}}\left(\alpha I+\beta \bar{\Lambda}_{\bar{r}}\right) \bar{P}^{2}\right)^{-1}$ are also triangular matrices.

Similarly, one can prove that the matrix $P \Gamma P^{-1}=\left(I_{2^{k}}+\tau^{2} \alpha P^{2}+\tau^{2} \beta P \Lambda_{\bar{r}} P\right)^{-1}$ is almost orthogonal similar to an upper triangular matrix $\bar{\Gamma}_{P}=W_{H}^{-1} P \Gamma P^{-1} W_{H}$ namely

$$
\bar{\Gamma}_{P}=\left(I_{2^{k}}+\frac{\tau^{2} \alpha}{2^{2 k}} \bar{P}^{2}+\frac{\tau^{2} \beta}{2^{2 k}} \bar{P} \bar{\Lambda}_{\bar{r}} \bar{P}\right)^{-1}
$$

Now we can rewrite the approximation of the discriminant $\Delta(\alpha, \beta)$ as

Lemma 4.2. If $\bar{\Gamma}$ and $\bar{\Gamma}_{P}$ are defined as in (4.1) and (4.2) respectively, then the approximation of the discriminant $\Delta(\alpha, \beta)$ can be written as

$$
\Delta(\alpha, \beta)=e^{T}\left(\bar{\Gamma}+\bar{\Gamma}_{P}\right) e_{2^{k}}
$$

where $e=\left[\begin{array}{llll}1 & 1 & \ldots & 1\end{array}\right]^{T}$ and $e_{2^{k}}=\left[\begin{array}{llll}0 & 0 & \ldots & 1\end{array}\right]^{T}$.

Proof. It follows from the claim (4.1)

$$
\begin{aligned}
\Delta(\alpha, \beta) & =e^{T} W_{H} W_{H}^{-1}\left(\Gamma+P \Gamma P^{-1}\right) W_{H} W_{H}^{-1} \bar{w}_{2^{k}}(\tau) \\
& =e^{T}\left(W_{H}^{-1}\left(\Gamma+P \Gamma P^{-1}\right) W_{H}\right) e_{2^{k}} \\
& =e^{T}\left(W_{H}^{-1} \Gamma W_{H}+W_{H}^{-1} P \Gamma P^{-1} W_{H}\right) e_{2^{k}} \\
& =e^{T}\left(\bar{\Gamma}+\bar{\Gamma}_{p}\right) e_{2^{k}}
\end{aligned}
$$

where $e=e_{1}^{T} W_{H}=\left[\begin{array}{llll}1 & 1 & \ldots & 1\end{array}\right]^{T}$ and $e_{2^{k}}=W_{H}^{-1} \bar{w}_{2^{k}}(\tau)=\left[\begin{array}{llll}0 & 0 & \ldots & 1\end{array}\right]^{T}$.

Notice that the discriminant approximation is the summation of the last column entries of the matrices $\bar{\Gamma}$ and $\bar{\Gamma}_{P}$ and it does not depend on the Walsh functions any longer.

At first sight the approximations (3.11) and (4.3) are almost the same, both of them have the same problem, they depend on the inverse of large dimensional matrices. But, the fundamental difference between both approximations is the structure of the matrices $\Gamma$ and $\bar{\Gamma}$, the former is a matrix full of numbers and seems to have no pattern at all, on the other hand, the latter is an upper triangular matrix and has a distinguishable pattern, see (5.1). Furthermore, thanks to the triangular form of $\bar{\Gamma}$ and $\bar{\Gamma}_{P}$ one can obtain their last column entries, which is done in the following section. 
Remark 4.3. As we had seen the discriminant is defined as $\Delta(\alpha, \beta)=x_{1}(\tau)+\dot{x}_{2}(\tau)$, if we set $t_{n}=n \frac{\tau}{2^{k}}$ instead of $\tau$, and change the notation of the discriminant so, the dependence on $t_{n}$ be explicit then, $\Delta(\alpha, \beta)$ may be written as $\Delta\left(\alpha, \beta, t_{n}\right)=x_{1}\left(t_{n}\right)+\dot{x}_{2}\left(t_{n}\right)$. From the approximation of the state transition matrix (3.10) it is clear that $\Delta\left(\alpha, \beta, t_{n}\right)=e^{T}\left(\bar{\Gamma}+\bar{\Gamma}_{p}\right) e_{n}$, where $e_{n}$ is equal to a $2^{k} \times 1$ vector of zeros but equal to one on the $n-t h$ entry, this follows from the fact $W_{H}^{-1} \bar{w}_{2^{k}}\left(t_{n}\right)=e_{n}$. So $\Delta\left(\alpha, \beta, t_{n}\right)$ gives us the value of the addition $x_{1}(t)+\dot{x}_{2}(t)$ at $t=t_{n}$, that $i s, e^{T}\left(\bar{\Gamma}+\bar{\Gamma}_{p}\right) e_{n}$ gives us the sampling of the addition $x_{1}(t)+\dot{x}_{2}(t)$. Thus, the matrices $\bar{\Gamma}$ and $\bar{\Gamma}_{P}$ may be seen as the discriminant sampling matrices.

\section{Explicit form of the discriminant $\Delta(\alpha, \beta)$}

In this part we give an explicit form of the discriminant $\Delta(\alpha, \beta)$ by removing the dependence on the inverse matrices $\bar{\Gamma}$ and $\bar{\Gamma}_{P}$, this is done thanks to their triangular form. First of all we write the matrices $\bar{\Gamma}$ and $\bar{\Gamma}_{P}$ in order to see the pattern they follow

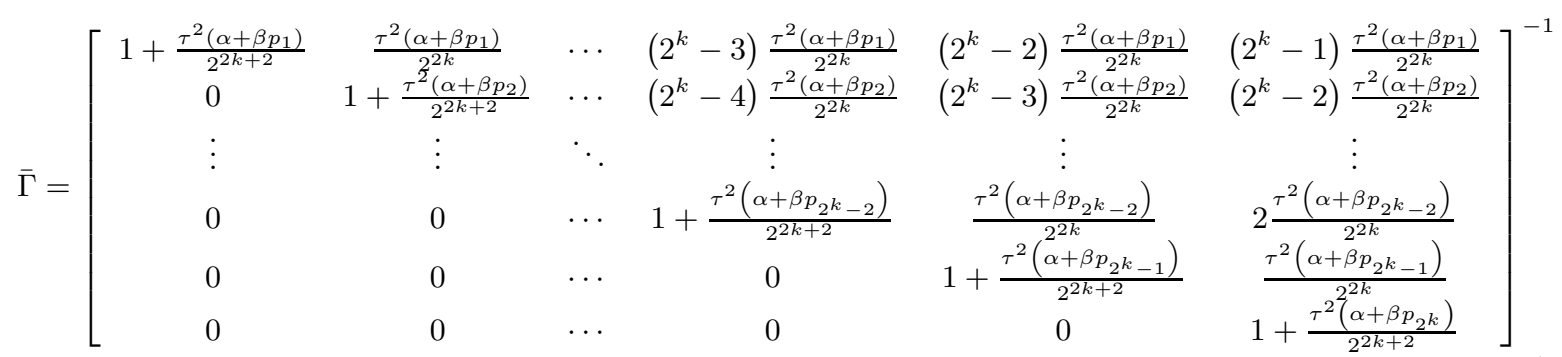

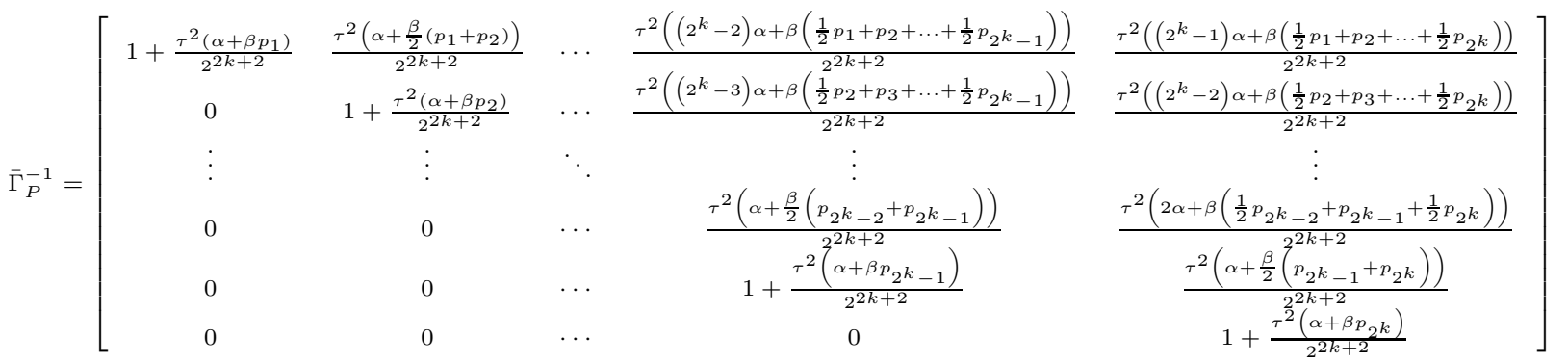

Now that we have explicitly written the matrices $\bar{\Gamma}$ and $\bar{\Gamma}_{p}$, we can deal with the non-singularity condition.

Remark 5.1. By simple inspection of (5.1) we can notice that the only chance for matrices $\bar{\Gamma}$ and $\bar{\Gamma}_{p}$ to be singular is that the function $q(t) \triangleq \alpha+\beta p(t)$ be large enough, in modulo, that some entry of the main diagonal be equal to zero. In other words for $\bar{\Gamma}$ and $\bar{\Gamma}_{p}$ to be singular the equality

$$
\alpha+\beta p_{n}=-\frac{2^{2 k+2}}{\tau^{2}}
$$

must be fulfilled for some $t_{n}=\frac{n \tau}{2^{k}}, n=1,2, \ldots, 2^{k}$. Remembering that $\bar{\Gamma}$ and $\bar{\Gamma}_{P}$ are $2^{k} \times 2^{k}$ real matrices and $2^{k}$ is the approximation order, (5.2) implies that for $\alpha, \beta$ and $p\left(t_{k}\right)$ small enough and large enough matrices the non-singularity is guaranteed.

As we said, in the previous section, the discriminant approximation (4.3) computation just requires the last column of the matrices $\bar{\Gamma}$ and $\bar{\Gamma}_{P}$, from now on we will call $b_{n}$ and $c_{n}\left(n=0,1, \ldots 2^{k}-1\right)$ to the $2^{k}-n$ entry of the last column of $\bar{\Gamma}$ and $\bar{\Gamma}_{P}$ respectively, i.e.

$$
\bar{\Gamma}=\left[\begin{array}{ccccc}
* & * & \cdots & * & b_{2^{k}-1} \\
0 & * & \cdots & * & b_{2^{k}-2} \\
\vdots & \vdots & \ddots & \vdots & \vdots \\
0 & 0 & \cdots & * & b_{1} \\
0 & 0 & \cdots & 0 & b_{0}
\end{array}\right] \text { and } \bar{\Gamma}_{P}=\left[\begin{array}{ccccc}
* & * & \cdots & * & c_{2^{k}-1} \\
0 & * & \cdots & * & c_{2^{k}-2} \\
\vdots & \vdots & \ddots & \vdots & \vdots \\
0 & 0 & \cdots & * & c_{1} \\
0 & 0 & \cdots & 0 & c_{0}
\end{array}\right]
$$


with this definitions the approximation of the discriminant can be written as

$$
\Delta(\alpha, \beta)=\sum_{n=0}^{2^{k}-1}\left(b_{n}+c_{n}\right)
$$

the Lemma in Appendix B.1 gives us a recursive method for obtaining the entries of the last column of a non-singular upper triangular matrix.

Now we are ready to state and prove the first of the two main results of this work, Theorem 5.2 gives us, in an explicit manner, the approximation of the discriminant $\Delta(\alpha, \beta)$ as a recursive summation, in other words, we eliminate the dependence on the inverse matrices $\bar{\Gamma}$ and $\bar{\Gamma}_{P}$.

\subsection{Eliminating the dependence on inverse matrices}

The following Lemma gives us a recursive method to obtain the coefficients $b_{n}$ and $c_{n}$, so the discriminant may be written as in (5.3).

Theorem 5.2. If the discriminant sampling matrices $\bar{\Gamma}$ and $\bar{\Gamma}_{P}$ are defined as in (4.1) and (4.2) respectively then, the entries $b_{n}$ and $c_{n}$ are:

$$
\begin{aligned}
b_{0} & =c_{0}=\frac{2^{2 k+2}}{2^{2 k+2}+\tau^{2}\left(\alpha+\beta p_{2^{k}}\right)} \\
b_{n} & =-\psi_{n} \xi_{n} \sum_{i=0}^{n-1} S_{i} \\
c_{n} & =-\psi_{n} \sum_{i=0}^{n-1}\left(c_{i} \sum_{j=i+1}^{n} \mu_{j}\right)
\end{aligned}
$$

for $n=1,2, \ldots, 2^{k}-1$ and with

$$
\begin{aligned}
S_{h} & =\sum_{i=0}^{h} b_{i} \\
Z_{h} & =\sum_{i=0}^{h} c_{i} \\
\psi_{h} & =\frac{4 \tau^{2}}{2^{2 k+2}+\tau^{2}\left(\alpha+\beta p_{2^{k}-h}\right)} \\
\xi_{h} & =\alpha+\beta p_{2^{k}-h} \\
\mu_{h} & =\alpha+\frac{\beta}{2}\left(p_{2^{k}-h}+p_{2^{k}-h+1}\right)
\end{aligned}
$$

And the discriminant approximation is

$$
\Delta(\alpha, \beta)=S_{2^{k-1}}+Z_{2^{k-1}}
$$

Proof. Defining $b_{n}, n=0,1, \ldots 2^{k}-1$, as the $2^{k}-n$ entry of the last column of $\bar{\Gamma}$ and by direct application of the Lemma in Appendix B.1 on matrix $\bar{\Gamma}$, eq. (5.1), and doing some simple algebraic operations one 
obtains

$$
\begin{aligned}
& b_{0}=\frac{2^{2 k+2}}{2^{2 k+2}+\tau^{2}\left(\alpha+\beta p_{2^{k}}\right)} \\
& b_{1}=-b_{0} \frac{\frac{\tau^{2}\left(\alpha+\beta p_{2^{k}-1}\right)}{2^{2 k}}}{1+\frac{\tau^{2}\left(\alpha+\beta p_{2^{k}-1}\right)}{2^{2 k+2}}} \\
& =4 \tau^{2} \frac{\alpha+\beta p_{2^{k}-1}}{2^{2 k+2}+\tau^{2}\left(\alpha+\beta p_{2^{k}-1}\right)}\left(-b_{0}\right) \\
& b_{2}=\frac{-b_{1} \frac{\tau^{2}\left(\alpha+\beta p_{2^{k}-2}\right)}{2^{2 k}}-2 b_{0} \frac{\tau^{2}\left(\alpha+\beta p_{2^{k}-2}\right)}{2^{2 k}}}{1+\frac{\tau^{2}\left(\alpha+\beta p_{2 k}\right)}{2^{2 k+2}}} \\
& =\frac{\frac{\tau^{2}\left(\alpha+\beta p_{2^{k}-2}\right)}{2^{2 k}}}{\frac{2^{2 k+2}+\tau^{2}\left(\alpha+\beta p_{2^{k}-2}\right)}{2^{2 k+2}}}\left(-b_{1}-2 b_{0}\right) \\
& =4 \tau^{2} \frac{\alpha+\beta p_{2^{k}-2}}{2^{2 k+2}+\tau^{2}\left(\alpha+\beta p_{2^{k}-2}\right)}\left(-b_{1}-2 b_{0}\right) \\
& b_{3}=4 \tau^{2} \frac{\alpha+\beta p_{2^{k}-3}}{2^{2 k+2}+\tau^{2}\left(\alpha+\beta p_{2^{k}-3}\right)}\left(-b_{2}-2 b_{1}-3 b_{0}\right) \\
& b_{n}=4 \tau^{2} \frac{\alpha+\beta p_{2^{k}-n}}{2^{2 k+2}+\tau^{2}\left(\alpha+\beta p_{2^{k}-n}\right)}\left(-b_{n-1}-\ldots-(n-1) b_{2}-n b_{0}\right)
\end{aligned}
$$

If we define

$$
\begin{aligned}
S_{h} & \triangleq \sum_{i=0}^{h} b_{i} \\
\psi_{h} & \triangleq \frac{4 \tau^{2}}{2^{2 k+2}+\tau^{2}\left(\alpha+\beta p_{2^{k}-h}\right)} \\
\xi_{h} \triangleq \alpha+\beta p_{2^{k}-h} & \triangleq \alpha
\end{aligned}
$$

then (5.7) becomes

$$
\begin{aligned}
b_{0} & =\frac{2^{2 k+2}}{2^{2 k+2}+\tau^{2}\left(\alpha+\beta p_{2^{k}}\right)} \\
b_{n} & =-\psi_{n} \xi_{n} \sum_{i=0}^{n-1} S_{i}
\end{aligned}
$$

for $n=1,2, \ldots 2^{k}-1$. By a similar procedure we can obtain the formulas for the coefficients $c_{n}$, see appendix C

$$
\begin{aligned}
& c_{0}=\frac{2^{2 k+2}}{2^{2 k+2}+\tau^{2}\left(\alpha+\beta p_{2^{k}}\right)} \\
& c_{n}=-\psi_{n} \sum_{i=0}^{n-1}\left(c_{i} \sum_{j=i+1}^{n} \mu_{j}\right) \\
& Z_{h} \triangleq \sum_{i=0}^{h} c_{i}
\end{aligned}
$$


where

$$
\mu_{h} \triangleq \alpha+\frac{\beta}{2}\left(p_{2^{k}-h}+p_{2^{k}-h+1}\right)
$$

for $n=1,2, \ldots 2^{k}-1, \psi_{h}$ is defined as in (5.9).

The last statement of the theorem follows from the equation (5.3) and the definitions of the summations $S_{h}$ and $Z_{h}$

Theorem 5.2 gives us a recursive method for obtaining the coefficients $b_{n}, c_{n}$ and the approximation of $\Delta(\alpha, \beta)$. Notice that if we define the function $q(t) \triangleq \alpha+\beta p(t)$ then, the coefficients will depend on the summation of $q\left(t_{n}\right)$ at $t_{n}=n \frac{\tau}{2^{k}}$ over a subset $J^{\prime}$ of $J=\left\{1,2, \ldots, 2^{k}\right\}$, i.e. $b_{n}$ and $c_{n}$ depend on $\sum_{n \in J^{\prime}} q\left(t_{n}\right)$ where $J^{\prime} \subset J$. Now, if we do $2^{k} \rightarrow \infty$ then $\frac{\tau}{2^{k}} \sum_{n \in J^{\prime}} q\left(t_{n}\right)$ behaves like a definite integral. And the next questions, arise: Could the coefficients $b_{n}$ and $c_{n}$ be written in terms of definite integrals? Moreover, Could the summation of the coefficients $b_{n}$ and $c_{n}$ be written as a summation of definite integrals? Next corollary gives the affirmative answer to the latter question.

Corollary 5.3. If the order $2^{k}$ of the approximation (5.6) is large enough so, $2^{k} \rightarrow \infty$ and $2^{2 k+2}>>$ $\tau^{2}\left(\alpha+\beta p_{2^{k}}\right)$ then, the summations of the first $n$ coefficients $b_{n}$ and $c_{n}, S_{n}=\sum_{i=0}^{n} b_{n}$ and $Z_{n}=\sum_{i=0}^{n} c_{n}$, are

$$
\begin{aligned}
& S_{0}=Z_{0} \approx 1 \\
& S_{n} \approx 1-\delta \sum_{i=0}^{n-1}\left[S_{i} \int_{\tau-(n+1) \delta}^{\tau-(i+1) \delta}(\alpha+\beta p(t)) d t\right] \\
& Z_{n} \approx 1-\delta\left[\sum_{i=0}^{n-1}(n-i) Z_{i} \int_{\tau-(i+2) \delta}^{\tau-(i+1) \delta}(\alpha+\beta p(t)) d t\right]
\end{aligned}
$$

Proof. As we are assuming that $2^{2 k+2}>>\tau^{2}\left(\alpha+\beta p_{2^{n}}\right)$ then (5.9) becomes

$$
\delta^{2} \triangleq \psi_{h} \approx \frac{\tau^{2}}{2^{2 k}}
$$

moreover

$$
b_{0}=c_{0} \approx 1
$$

For the first part of the corollary we must notice that the summation $S_{n}=\sum_{i=0}^{n} b_{n}$ can be written as

$$
S_{n} \approx 1-\delta^{2} \sum_{i=0}^{n-1}\left[S_{i} \sum_{j=i+1}^{n} \xi_{j}\right]
$$

it follows since

$$
\begin{aligned}
b_{0} \approx & 1 \\
b_{1} \approx & -\delta^{2} \xi_{1} S_{0} \\
b_{2} \approx & -\delta^{2} \xi_{2}\left(S_{0}+S_{1}\right) \\
& \vdots \\
b_{n} \approx & -\delta^{2} \xi_{n}\left(S_{0}+S_{1}+\ldots+S_{n-2}+S_{n-1}\right)
\end{aligned}
$$

adding the coefficients $b_{0}$ to $b_{n}$ and grouping terms we have

$$
\sum_{i=0}^{n} b_{n} \approx 1-\delta^{2}\left[S_{0}\left(\xi_{1}+\ldots+\xi_{n}\right)+S_{1}\left(\xi_{2}+\ldots+\xi_{n}\right)+\ldots+S_{n-1}\left(\xi_{n}\right)\right]
$$


thus (5.15) follows.

From the definition of $\xi_{h}$ and $\delta$, equations (5.10) and (5.14) respectively, one can notice that for $\ell<n$

$$
\lim _{\delta \rightarrow 0} \delta \sum_{i=\ell}^{n} \xi_{i} \approx \int_{\tau-(n+1) \delta}^{\tau-(\ell+1) \delta}(\alpha+\beta p(t)) d t
$$

then, we can rewrite (5.15) as a summation that depends on integrals

$$
S_{n} \approx 1-\delta \sum_{i=0}^{n-1}\left[S_{i} \int_{\tau-(n+1) \delta}^{\tau-(i+1) \delta}(\alpha+\beta p(t)) d t\right]
$$

For the second part of the corollary, we directly apply the definite integral definition to (5.5) and one obtains

$$
\begin{aligned}
c_{n} & \approx-\delta^{2} \sum_{i=0}^{n-1}\left(c_{i} \sum_{j=i+1}^{n} \mu_{j}\right) \\
& =-\delta \sum_{j=0}^{n-1}\left(c_{j} \int_{\tau-(n+1) \delta}^{\tau-(j+1) \delta}(\alpha+\beta p(t)) d t\right)
\end{aligned}
$$

thus, if $Z_{n}=\sum_{i=0}^{n} c_{n}$

$$
\begin{aligned}
Z_{0} \approx & 1 \\
Z_{1} \approx & 1-\delta\left(c_{0} \int_{\tau-2 \delta}^{\tau-\delta}(\alpha+\beta p(t)) d t\right) \\
Z_{2} \approx & 1-\delta\left(c_{0}^{\tau-1 \delta}(\alpha+\beta p(t)) d t+c_{0} \int_{\tau-3 \delta}^{\tau-1 \delta}(\alpha+\beta p(t)) d t+c_{1} \int_{\tau-3 \delta}^{\tau-2 \delta}(\alpha+\beta p(t)) d t\right) \\
= & 1-\delta\left(2 c_{0} \int_{\tau-2 \delta}^{\tau-1 \delta}(\alpha+\beta p(t)) d t+\left(c_{0}+c_{1}\right) \int_{\tau-3 \delta}^{\tau-2 \delta}(\alpha+\beta p(t)) d t\right) \\
Z_{3} \approx & 1-\delta\left(3 c_{0} \int_{\tau-2 \delta}^{\tau-1 \delta}(\alpha+\beta p(t)) d t+2\left(c_{0}+c_{1}\right) \int_{\tau-3 \delta}(\alpha+\beta p(t)) d t+\left(c_{0}+c_{1}+c_{2}\right)\right. \\
\tau & (\alpha)) d t) \\
& \vdots \\
Z_{n} \approx & 1-\delta\left[\sum_{i=0}^{n-1}(n-i) Z_{i} \int_{\tau-(i+2) \delta}(\alpha+\beta p(t)) d t\right]
\end{aligned}
$$

Corollary 5.3 not only gives us a recursive method to obtain the summation of the coefficients $b_{n}$ and $c_{n}$, but, by doing some considerations, it transforms the dependence of the "discrete" approximation of Theorem 5.2 on some discrete values of the excitation function $p(t)$ into a dependence on the definite integral of the continuous function $p(t)$. We should notice that the new expressions of the summations of the coefficients $b_{n}$ 
and $c_{n}$ ( $S_{n}$ and $Z_{n}$ respectively) depend on $\delta$ and on the summation of a large number of definite integrals, so it seems that the discriminant approximation $\Delta(\alpha, \beta) \approx S_{2^{k}-1}+Z_{2^{k}-1}$ can be reduced even more.

Now we are ready to prove the second main result of this work. In the next Theorem we give an alternative proof of the discriminant approximation obtained by Lyapunov in his outstanding work [3] , see Section 1. This new proof is based on Theorem 5.2 and corollary [5.3] and is completely independent of the proof made by Lyapunov.

Theorem 5.4. If $\Delta(\alpha, \beta)$ is the discriminant of a second order periodic differential equation

$$
\ddot{x}+q(t) x=0, \quad q(\tau+t)=q(t)
$$

then, $\Delta(\alpha, \beta)$ can be expressed as an alternating series

$$
\Delta(\alpha, \beta)=2-A_{1}+A_{2}+\ldots+(-1)^{n} A_{n}
$$

where the constants $A_{n} n=1,2, \ldots, 2^{k}-1$ are defined as the multiple integrals

$$
\begin{aligned}
A_{0}= & 2, \quad A_{1}=\tau \int_{0}^{\tau} q\left(t_{1}\right) d t_{1} \\
A_{2}= & \int_{0}^{\tau} d t_{1} \int_{0}^{t_{1}}\left(\tau-t_{1}+t_{2}\right)\left(t_{1}-t_{2}\right) q\left(t_{1}\right) q\left(t_{2}\right) d t_{2} \\
& \vdots \\
A_{n}= & \int_{0}^{\tau} d t_{1} \int_{0}^{t_{1}} d t_{2} \ldots \int_{0}^{t_{n-1}}\left(\tau-t_{1}+t_{n}\right)\left(t_{1}-t_{2}\right) \ldots\left(t_{n-1}-t_{n}\right) q\left(t_{1}\right) q\left(t_{2}\right) \ldots q\left(t_{n}\right) d t_{n}
\end{aligned}
$$

Proof. We know that $\Delta(\alpha, \beta)=S_{2^{k}-1}+Z_{2^{k}-1}$, moreover, corollary 5.3 gives us recursive formulas to obtain $S_{n}$ and $Z_{n}$. The proof of this theorem is based on rewriting the formulas for $S_{n}$ and $Z_{n}$, this rewriting must replace the recursion by an expansion in terms of powers of the parameter $\delta=\frac{\tau}{2^{k}}$. If we define

$$
\mathbb{I}_{n, m} \triangleq \int_{\tau-n \delta}^{\tau-m \delta} q\left(t_{1}\right) d t_{1}
$$

then, $S_{n}$ and $Z_{n}$, equation (5.12) and (5.13) respectively, are

$$
\begin{aligned}
S_{0} & =Z_{0}=1 \\
S_{n} & =1-\delta \sum_{i=0}^{n-1}\left[S_{i} \mathbb{I}_{n+1, i+1}\right] \\
Z_{n} & =1-\delta\left[\sum_{i=0}^{n-1}(n-i) Z_{i} \mathbb{I}_{i+2, i+1}\right]
\end{aligned}
$$

Following the formula for $S_{n}$ and $Z_{n}$ and grouping terms of powers of $\delta$ one obtains, see Lemma in Appendix D.1

$$
\begin{aligned}
& S_{n}= 1-\delta \sum_{i=1}^{n} \mathbb{I}_{n+1, i}+\delta^{2} \sum_{i=2}^{n} \mathbb{I}_{n+1, i} \sum_{j=1}^{i-1} \mathbb{I}_{i, j}-\delta^{3} \sum_{i=3}^{n} \mathbb{I}_{n+1, i} \sum_{j=1}^{i-1} \mathbb{I}_{i, j} \sum_{l=1}^{j} \mathbb{I}_{j, l}+\ldots \\
& \ldots+(-1)^{\bar{n}} \delta^{\bar{n}} \sum_{i=\bar{n}}^{n} \mathbb{I}_{n+1, i} \sum_{j=1}^{i-1} \mathbb{I}_{i, j} \sum_{l=1}^{j} \mathbb{I}_{j, l} \ldots \sum_{y=1}^{x} \mathbb{I}_{x, y} \\
& Z_{n}=1-\delta \sum_{i=2}^{n+1} \mathbb{I}_{i, 1}+\delta^{2} \sum_{i=2}^{n} \mathbb{I}_{i, 1} \sum_{j=i+1}^{n+1} \mathbb{I}_{j, i}-\delta^{3} \sum_{i=2}^{n-1} \mathbb{I}_{i, 1} \sum_{j=i+1}^{n} \mathbb{I}_{j, i} \sum_{l=j+1}^{n+1} \mathbb{I}_{l, j}+\ldots \\
& \ldots+(-1)^{\bar{n}} \delta^{\bar{n}} \sum_{i=2}^{n+2-\bar{n}} \mathbb{I}_{i, 1} \sum_{j=i+1}^{n+3-\bar{n}} \mathbb{I}_{j, i} \ldots \sum_{y=x+1}^{n+1} \mathbb{I}_{y, x}
\end{aligned}
$$


As we have said the discriminant $\Delta(\alpha, \beta)$ is approximately equal to the addition of $S_{2^{k}-1}$ and $Z_{2^{k}-1}$, then $\Delta(\alpha, \beta)$ may be written as an expansion of powers of $\delta$. If we define the coefficients $A_{n}, n=0,1,2, \ldots, 2^{k}-1$, as the terms associated to the $n-t h$ power of $\delta$, i.e.

$$
\begin{gathered}
A_{0}=2 \\
A_{1}=\delta\left(\sum_{i=1}^{2^{k}-1} \mathbb{I}_{2^{k}, i}+\sum_{i=2}^{2^{k}} \mathbb{I}_{i, 1}\right) \\
A_{2}=\delta^{2}\left[\sum_{i=2}^{2^{k}-1}\left(\mathbb{I}_{2^{k}, i} \sum_{j=1}^{i-1} \mathbb{I}_{i, j}\right)+\sum_{i=2}^{2^{k}-1}\left(\mathbb{I}_{i, 1} \sum_{j=i+1}^{2^{k}-1+1} \mathbb{I}_{j, i}\right)\right] \\
A_{3}=\delta^{3}\left[\sum_{i=3}^{n}\left(\mathbb{I}_{n+1, i} \sum_{j=1}^{i-1} \mathbb{I}_{i, j} \sum_{l=1}^{j} \mathbb{I}_{j, l}\right)+\sum_{i=2}^{n-1}\left(\mathbb{I}_{i, 1} \sum_{j=i+1}^{n} \mathbb{I}_{j, i} \sum_{l=j+1}^{n+1} \mathbb{I}_{l, j}\right)\right] \\
A_{n}=\delta^{n}\left[\sum_{i=n}^{2^{k}-1}\left(\mathbb{I}_{2^{k}, i} \sum_{j=1}^{i-1} \mathbb{I}_{i, j} \sum_{l=1}^{j} \mathbb{I}_{j, l} \ldots \sum_{y=1}^{x} \mathbb{I}_{x, y}\right)+\sum_{i=2}^{2^{k}+1-n}\left(\mathbb{I}_{i, 1} \sum_{j=i+1}^{2^{k}+2-n} \mathbb{I}_{j, i} \ldots \sum_{y=x+1}^{2^{k}} \mathbb{I}_{y, x}\right)\right]
\end{gathered}
$$

then the discriminant is

$$
\Delta(\alpha, \beta)=A_{0}-A_{1}+A_{2}+\ldots+(-1)^{n} A_{n}
$$

Notice that the coefficients $A_{n}$ in (5.16) can be rewritten as the multiple integrals

$$
\begin{aligned}
A_{1}= & \int_{0}^{\tau-\delta} \int_{0}^{t_{1}} q\left(t_{2}\right) d t_{2} d t_{1}+\int_{0}^{\tau-\delta} \int_{t_{1}}^{\tau-\delta} q\left(t_{2}\right) d t_{2} d t_{1} \\
A_{2}= & \int_{\delta}^{\tau-\delta} \int_{0}^{t_{1}} q\left(t_{2}\right) d t_{2} \int_{t_{1}}^{\tau-\delta} \int_{t_{1}}^{t_{3}} q\left(t_{4}\right) d t_{4} d t_{3} d t_{1}+\int_{\delta}^{\tau-\delta} \int_{t_{1}}^{\tau-\delta} q\left(t_{2}\right) d t_{2} \int_{0}^{t_{1}} \int_{t_{3}}^{t_{1}} q\left(t_{4}\right) d t_{4} d t_{3} d t_{1} \\
A_{3}= & \int_{\delta}^{\tau-3 \delta} \int_{0}^{t_{1}} q\left(t_{2}\right) d t_{2} \int_{t_{1}}^{\tau-\delta} \int_{t_{1}}^{t_{3}} q\left(t_{4}\right) d t_{4} \int_{t_{3}}^{\tau-\delta} \int_{t_{3}}^{t_{5}} q\left(t_{6}\right) d t_{6} d t_{5} d t_{3} d t_{1} \\
& +\int_{2 \delta}^{\tau-\delta} \int_{t_{1}}^{\tau-\delta} q\left(t_{2}\right) d t_{2} \int_{\delta}^{t_{1}} \int_{t_{3}}^{t_{1}} q\left(t_{4}\right) d t_{4} \int_{0}^{t_{3}} \int_{t_{5}}^{t_{3}} q\left(t_{6}\right) d t_{6} d t_{5} d t_{3} d t_{1} \\
& \vdots \\
A_{n}= & \int_{\delta}^{\tau-n \delta} \int_{0}^{t_{1}} q\left(t_{2}\right) \ldots \int_{t_{2 n-5}}^{\tau-\delta} \int_{t_{2 n-5}}^{t_{2 \bar{n}-3}} q\left(t_{2 n-2}\right) \int_{t_{2 n-3}-\delta}^{\tau-3} \int_{t_{2 n-3}}^{t_{2 n-1}} q\left(t_{2 n}\right) d t_{2 n} d t_{2 n-1} \ldots d t_{2} d t_{1} \\
& +\int_{2 \delta}^{\tau-\delta} \int_{t_{1}}^{\tau-\delta} q\left(t_{2}\right) \ldots \int_{\delta}^{t_{2 n-5}} \int_{t_{2 n-3}}^{t_{2 n-5}} q\left(t_{2 n-2}\right) \int_{0}^{t_{2 n-3}} \int_{t_{2 n-1}}^{t_{2 n-3}} q\left(t_{2 n}\right) d t_{2 n} d t_{2 n-1} \ldots d t_{2} d t_{1}
\end{aligned}
$$


since $2^{k} \rightarrow \infty$ then, $\delta \rightarrow 0$ and for values of $\bar{n}$ such that $\bar{n} \delta \rightarrow 0$ then

$$
\begin{aligned}
A_{1}= & \int_{0}^{\tau} \int_{0}^{t_{1}} q\left(t_{2}\right) d t_{2} d t_{1}+\int_{0}^{\tau} \int_{t_{1}}^{\tau} q\left(t_{2}\right) d t_{2} d t_{1} \\
A_{2}= & \int_{0}^{\tau} \int_{0}^{t_{1}} \int_{t_{1}}^{\tau} \int_{t_{1}}^{t_{3}} q\left(t_{2}\right) q\left(t_{4}\right) d t_{4} d t_{3} d t_{2} d t_{1}+\int_{0}^{\tau} \int_{t_{1}}^{\tau} \int_{0}^{t_{1}} \int_{t_{3}}^{t_{1}} q\left(t_{2}\right) q\left(t_{4}\right) d t_{4} d t_{3} d t_{2} d t_{1} \\
A_{3}= & \int_{0}^{\tau} \int_{0}^{t_{1}} \int_{t_{1}}^{\tau} \int_{t_{1}}^{t_{3}} \int_{t_{3}}^{\tau} \int_{t_{3}}^{t_{5}} q\left(t_{2}\right) q\left(t_{4}\right) q\left(t_{6}\right) d t_{6} d t_{5} d t_{4} d t_{3} d t_{2} d t_{1} \\
& +\int_{0}^{\tau} \int_{t_{1}}^{\tau} \int_{0}^{t_{1}} \int_{t_{3}}^{t_{1}} \int_{0}^{t_{3}} \int_{t_{5}}^{t_{3}} q\left(t_{2}\right) q\left(t_{4}\right) q\left(t_{6}\right) d t_{6} d t_{5} d t_{4} d t_{3} d t_{2} d t_{1} \\
& \vdots \\
A_{\bar{n}}= & \int_{0}^{\tau} \int_{0}^{t_{1}} \ldots \int_{t_{2 \bar{n}-5}}^{\tau} \int_{t_{2 \bar{n}-5}}^{t_{2 \bar{n}-3}} \int_{t_{2 \bar{n}-3}}^{\tau} \int_{t_{2 \bar{n}-3}}^{t_{2 \bar{n}-1}} p\left(t_{2}\right) \ldots p\left(t_{2 \bar{n}-2}\right) p\left(t_{2 \bar{n}}\right) d t_{2 \bar{n}} d t_{2 \bar{n}-1} \ldots d t_{2} d t_{1} \\
& +\int_{0}^{\tau} \int_{t_{1}}^{\tau} \ldots \int_{0}^{t_{2 \bar{n}}-5} \int_{t_{2 \bar{n}-3}}^{t_{2 \bar{n}}-5} \int_{0}^{t_{2 \bar{n}-3}} \int_{t_{2 \bar{n}-1}}^{t_{2 \bar{n}-3}} p\left(t_{2}\right) \ldots p\left(t_{2 \bar{n}-2}\right) p\left(t_{2 \bar{n}}\right) d t_{2 \bar{n}} d t_{2 \bar{n}-1} \ldots d t_{2} d t_{1}
\end{aligned}
$$

It remains to be proven that the coefficients $A_{n}$ are equal to the coefficients obtained by Lyapunov, which is simply done by rearranging the integration variables, see Lemma in Appendix D.2 for the first three coefficients,

$$
\begin{aligned}
& A_{1}=\tau \int_{0}^{\tau} q\left(t_{2}\right) d t_{2} \\
& A_{2}=\int_{0}^{\tau} \int_{0}^{t_{2}}\left(\tau-t_{2}+t_{1}\right)\left(t_{2}-t_{1}\right) q\left(t_{1}\right) q\left(t_{2}\right) d t_{1} d t_{2} \\
& A_{3}=\int_{0}^{\tau} \int_{0}^{t_{6}} \int_{0}^{t_{4}}\left(\tau-t_{6}+t_{2}\right)\left(t_{6}-t_{4}\right)\left(t_{4}-t_{2}\right) q\left(t_{2}\right) q\left(t_{4}\right) q\left(t_{6}\right) d t_{2} d t_{4} d t_{6}
\end{aligned}
$$

the general term is

$$
A_{n}=\int_{0}^{\tau} d t_{1} \int_{0}^{t_{1}} d t_{2} \ldots \int_{0}^{t_{\bar{n}-1}}\left(\tau-t_{1}+t_{n}\right)\left(t_{1}-t_{2}\right) \ldots\left(t_{n-1}-t_{n}\right) q\left(t_{1}\right) q\left(t_{2}\right) \ldots q\left(t_{n}\right) d t_{n}
$$

thus, the theorem follows.

Notice that the coefficients $A_{n}$ of Theorem 5.4 are equal to the coefficients of the Lyapunov approximation, see section 1 , but for a factor $\frac{1}{2}$, this difference is because we consider the discriminant as $\Delta(\alpha, \beta)=x_{1}(\tau)+\dot{x}_{2}(\tau)$ and Lyapunov defined its characteristic constant as $A=\frac{1}{2}\left(x_{1}(\tau)+\dot{x}_{2}(\tau)\right)$.

Remark 5.5. Since the approximation made by Lyapunov depends on multiple integrals (the number of multiple integrals that one has to calculate is equal to the sub index of each coefficient $A_{n}$ ), it is very hard to compute. Nevertheless, Theorem 5.2 give us a recursive method for obtaining the approximation of $\Delta(\alpha, \beta)$.

By doing $2^{k} \rightarrow \infty$, we have taken the approximation of Theorem 5.2, which depends on some discrete values of the function $p(t)$ and we have transformed it to an approximation that depends on definite integrals of the excitation function $p(t)$. So, one can say that Theorem 5.2 may be seen as a "discrete" form of the discriminant approximation made by Lyapunov.

\section{Numerical calculation of the discriminant approximation $\Delta(\alpha, \beta)$}

As we know the discriminant $\Delta(\alpha, \beta)$ plays a very important role in the determination of the stability zones of linear periodic differential equations. By using Theorem 5.2 we are able to compute an approximation of the discriminant $\Delta(\alpha, \beta)$ at each point of the $\alpha-\beta$ plane and then use the stability conditions 
of Theorem 2.4 to find the stable zones $(|\Delta(\alpha, \beta)|<2)$, unstable zones $(|\Delta(\alpha, \beta)|>2)$ or the transition curves $(|\Delta(\alpha, \beta)|=2)$ of any Hill equation.

We must notice that the discriminant $\Delta(\alpha, \beta)$ defines a manifold $(\alpha, \beta, \Delta(\alpha, \beta))$ in $\mathbb{R}^{3}$ which contains all the stability properties of the periodic differential equation. It is not so hard to see that the projection of the intersection between the manifold $(\alpha, \beta, \Delta(\alpha, \beta))$ and the surfaces $\operatorname{surf}_{1}=\{(\alpha, \beta, z) \mid \forall \alpha, \beta \in \mathbb{R}, z=2\}$ and $\operatorname{surf}_{2}=\{(\alpha, \beta, z) \mid \forall \alpha, \beta \in \mathbb{R}, z=-2\}$, in the $\alpha-\beta$ plane, are the transition curves of a Hill equation, see Fig. 2.

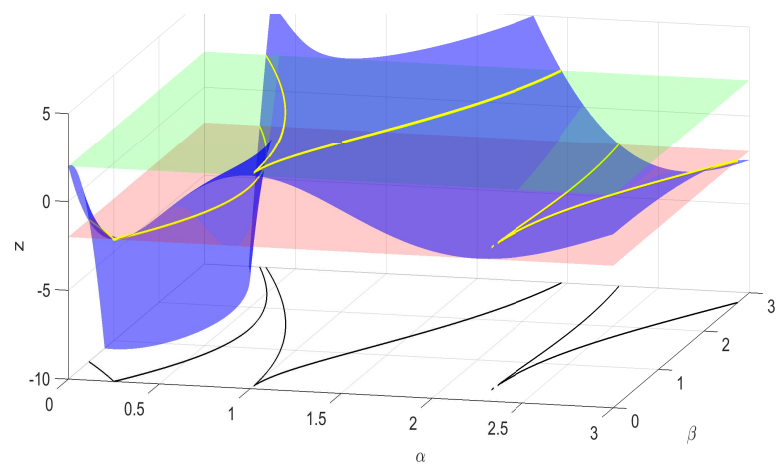

Figure 2: Discriminant approximation manifold in blue, $\operatorname{surf}_{1}$ and $\operatorname{surf} f_{2}$ in red and green respectively, yellow lines represents the intersection between $\Delta(\alpha, \beta)$ and $\operatorname{surf}_{1}$ or $\operatorname{surf} f_{2}$, transition curves in black.

Figure 3 shows the approximation, for two different approximation orders, and the actual transition curves of the periodic differential equation

$$
\ddot{x}+(\alpha+\beta(\cos (t)+\cos (2 t))) x=0
$$

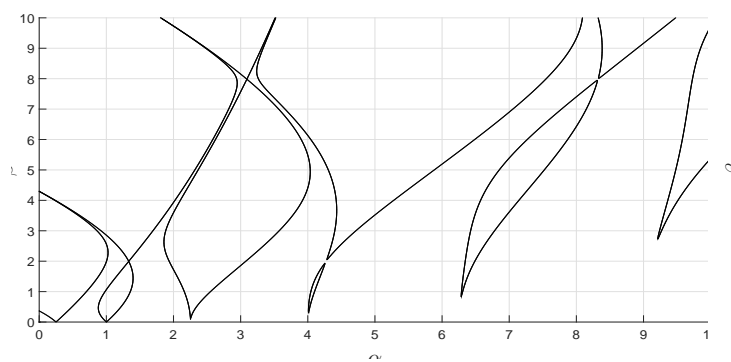

(a)

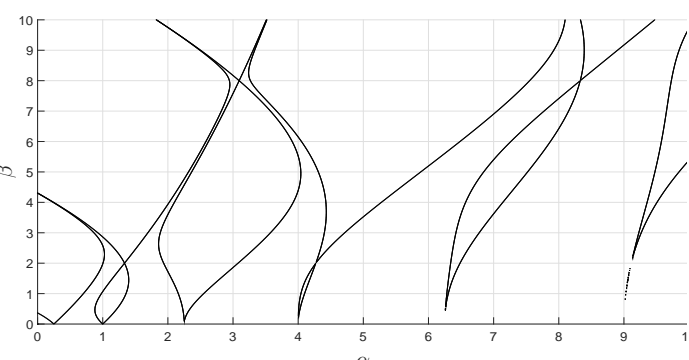

(b)

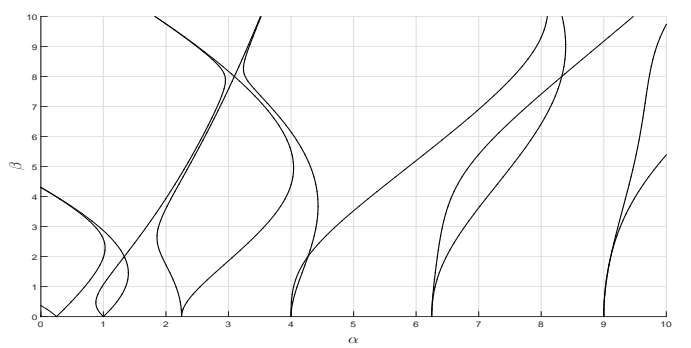

(c)

Figure 3: a) Transition curves approximation of order $2^{10}$, b) Transition curves approximation of order $2^{15}$ c) Actual transition curves of 6.1 . 
Notice that, as it was expected, the approximation of the transition curves of order $2^{15}$ is better than the one with order $2^{10}$, nevertheless, the latter gives us an idea of the shape of the actual transition curves.

As a final remark we must say that the approximation here developed, was obtained thanks to the Walsh function properties. It is true that all orthogonal series, such as Fourier series, Bessel functions, share some common properties such as the possibility of obtaining integral or differential operators or the closeness under the multiplication, that is, if $f(t)$ and $g(t)$ belong to an infinite set of orthogonal series the product $f(t) g(t)$ may be represented in terms of the infinite set of orthogonal series. But, Walsh functions have some extra properties, that just a few other sets of orthogonal series have, for example the multiplication of two Walsh functions belonging to a finite set may be expressed in terms of the finite set elements. Another property is the one presented in Lemma 3.1 see section 3, which allows us to post factorize the vector of Walsh function $\bar{w}_{2^{k}}$ given the multiplication $\bar{w}_{2^{k}} \bar{w}_{2^{k}}^{T} \gamma$; this property made it possible to obtain the state transition matrix approximation in (3.10). And, finally the almost orthogonal similarity of the upper triangular matrices: $\bar{\Lambda}_{\bar{r}}=W_{H}^{-1} \Lambda_{\bar{r}} W_{H}$ ( $p(t)$ sampling matrix), $\bar{P}=W_{H} P W_{H}$ (integration operator), $\bar{\Gamma}=W_{H}^{-1} \bar{\Gamma} W_{H}$ and $\bar{\Gamma}_{P}=W_{H}^{-1} \bar{\Gamma}_{P} W_{H}$ (discriminant sampling matrices), which is the most fundamental property for the development of the approximation (5.6), see Theorem 5.2 .

\section{Conclusion}

In section 5 we have given an alternative proof of the discriminant approximation $\Delta(\alpha, \beta)$ made by Lyapunov in [3] and studied in depth in [5]. This new proof is based on some properties of Walsh functions and basic definitions and properties of multiple integrals. In spite of being one of the most known approximations, the Lyapunov discriminant approximation is very difficult to obtain since it is necessary to calculate a very large number of multiple integrals. In this work, the alternative is easily programmed and allows us to have a computational approach to the discriminant approximation $\Delta(\alpha, \beta)$ to any desired accuracy.

We give a new approximation of $\Delta(\alpha, \beta)$, this is a recursive summation and it only depends on the evaluation of the excitation function at the time $t_{n} \in[0, \tau], n=0,1,2, \ldots 2^{k}-1$, where $2^{k}$ is the order of the approximation, see Theorem 5.2. First, the new approximation of $\Delta(\alpha, \beta)$ was obtained by means of Walsh functions, $\Delta(\alpha, \beta)$ depended on two large dimensional inverse matrices $\Gamma$ and $\Gamma_{p}$, see (4.1), the dependence of $\Delta(\alpha, \beta)$ on Walsh functions was then eliminated by a similarity transformation of $\Gamma$ and $\Gamma_{p}$, thus the new form of $\Delta(\alpha, \beta)$ depended on the summation of the last column entries of the two large dimensional triangular inverse matrices $\bar{\Gamma}$ and $\bar{\Gamma}_{p}$, see equation (4.3) on Lemma 4.2. Then, the last column entries of $\bar{\Gamma}$ and $\bar{\Gamma}_{p}$ were calculated and the recursive summation was obtained.

The new approximation, Theorem 5.2. may be seen as a "discrete" form of the discriminant approximation made by Lyapunov. This approximation is easy to implement on a computer. The latter is important because the accuracy of the approximation depends on the number of recursions, i.e. as $2^{k} \rightarrow \infty$ the accuracy of the method will be better.

\section{Appendix A. Walsh function vector permutation matrix}

Next Lemma states that the columns of the symmetric matrix defined as $M_{2^{k}}=\bar{w}_{2^{k}} \bar{w}_{2^{k}}^{T}$ are the permutations of the vector $\bar{w}_{2^{k}}$. The proof is based on the fact that if $w_{n}(t)$ and $w_{m}(t)$ belong to a finite set of Walsh functions $\left\{w_{0}(t), w_{1}(t), \ldots, w_{2^{k}-1}(t)\right\}$ then, the multiplication $w_{n}(t) w_{m}(t)$ belongs to the same finite set to which the functions $w_{n}(t)$ and $w_{m}(t)$ belong.

Lemma Appendix A.1. Let $\bar{w}_{2^{k}}(t)$ be the $2^{k} \times 1$ vector of first $2^{k}$ Walsh functions $w_{n}(t), n=0,1, \ldots, 2^{k}-$ 1 , and $M_{2^{k}}=\bar{w}_{2^{k}} \bar{w}_{2^{k}}^{T}$ then the columns of the matrix $M$ are permutations of the entries of the vector $\bar{w}_{2^{k}}(t)$ and it can be written as

$$
M_{2^{k}}(t)=\left[\bar{w}_{2^{k}}(t), \Lambda_{1}^{\left(2^{k}\right)} \bar{w}_{2^{k}}(t), \ldots, \Lambda_{2^{k}-1}^{\left(2^{k}\right)} \bar{w}_{2^{k}}(t)\right]
$$


where

$$
\begin{aligned}
\Lambda_{i}^{\left(2^{k}\right)} & =\left[\begin{array}{cc}
\Lambda_{i}^{\left(2^{k} / 2\right)} & 0_{\left(2^{k} / 2\right)} \\
0_{\left(2^{k} / 2\right)} & \Lambda_{i}^{\left(2^{k} / 2\right)}
\end{array}\right] \\
\Lambda_{i+\left(2^{k} / 2\right)}^{\left(2^{k}\right)} & =\left[\begin{array}{ll}
0_{\left(2^{k} / 2\right)} & \Lambda_{i}^{\left(2^{k} / 2\right)} \\
\Lambda_{i}^{\left(2^{k} / 2\right)} & 0_{\left(2^{k} / 2\right)}
\end{array}\right] \\
\Lambda_{0}^{\left(2^{k}\right)} & =I_{2^{k}} \\
\Lambda_{i}^{\left(2^{k}\right)} & \in R^{2^{k} \times 2^{k}}
\end{aligned}
$$

The proof may be found in 24]. For didactic purposes, let us take $k=2$ and since $w_{n}(t) w_{m}(t)=$ $w_{n \oplus m}(t)$, where $\oplus$ refers the the no-carry modulo- 2 addition, the matrix $M_{4}$ is

$$
M_{4}(t)=\left[\begin{array}{llll}
w_{0}(t) & w_{1}(t) & w_{2}(t) & w_{3}(t) \\
w_{1}(t) & w_{0}(t) & w_{3}(t) & w_{2}(t) \\
w_{2}(t) & w_{3}(t) & w_{0}(t) & w_{1}(t) \\
w_{3}(t) & w_{2}(t) & w_{1}(t) & w_{0}(t)
\end{array}\right]
$$

from where it can be seen that the second, third and forth columns of $M_{4}$ are permutations of the entries of the first column, even more the second column can be rewritten as

$$
\left[\begin{array}{l}
w_{1}(t) \\
w_{0}(t) \\
w_{3}(t) \\
w_{2}(t)
\end{array}\right]=\left[\begin{array}{llll}
0 & 1 & 0 & 0 \\
1 & 0 & 0 & 0 \\
0 & 0 & 0 & 1 \\
0 & 0 & 1 & 0
\end{array}\right]\left[\begin{array}{l}
w_{0}(t) \\
w_{1}(t) \\
w_{2}(t) \\
w_{3}(t)
\end{array}\right]
$$

or simply $\Lambda_{1} \bar{w}_{4}(t)$, the third column is

$$
\left[\begin{array}{l}
w_{2}(t) \\
w_{3}(t) \\
w_{0}(t) \\
w_{1}(t)
\end{array}\right]=\left[\begin{array}{llll}
0 & 0 & 1 & 0 \\
0 & 0 & 0 & 1 \\
1 & 0 & 0 & 0 \\
0 & 1 & 0 & 0
\end{array}\right]\left[\begin{array}{l}
w_{0}(t) \\
w_{1}(t) \\
w_{2}(t) \\
w_{3}(t)
\end{array}\right]
$$

or simply $\Lambda_{2} \bar{w}_{4}(t)$, and the fourth column is

$$
\left[\begin{array}{l}
w_{3}(t) \\
w_{2}(t) \\
w_{1}(t) \\
w_{0}(t)
\end{array}\right]=\left[\begin{array}{llll}
0 & 0 & 0 & 1 \\
0 & 0 & 1 & 0 \\
0 & 1 & 0 & 0 \\
1 & 0 & 0 & 0
\end{array}\right]\left[\begin{array}{l}
w_{0}(t) \\
w_{1}(t) \\
w_{2}(t) \\
w_{3}(t)
\end{array}\right]
$$

or simply $\Lambda_{3} \bar{w}_{4}(t)$, so

$$
M_{4}(t)=\left[\bar{w}_{4}(t), \Lambda_{1} \bar{w}_{4}(t), \Lambda_{2} \bar{w}_{4}(t), \Lambda_{3} \bar{w}_{4}(t)\right]
$$

which is consistent with the above mentioned lemma.

\section{Appendix B. Obtaining the last column of an upper triangular inverse matrix}

In this part, the last column entries of a non-singular upper triangular matrix, are obtained as a recursive summation. 
Let $U$ be the $n \times n$ real non-singular matrix

$$
U=\left[\begin{array}{cccccc}
u_{1,1} & u_{1,2} & \cdots & u_{1, n-2} & u_{1, n-1} & u_{1, n} \\
0 & u_{22} & \cdots & u_{2, n-2} & u_{2, n-1} & u_{2, n} \\
\vdots & \vdots & \ddots & \vdots & \vdots & \vdots \\
0 & 0 & \cdots & u_{n-2, n-2} & u_{n-2, n-1} & u_{n-2, n} \\
0 & 0 & \cdots & 0 & u_{n-1, n-1} & u_{n-1, n} \\
0 & 0 & \cdots & 0 & 0 & u_{n, n}
\end{array}\right]
$$

and define its inverse as

$$
U^{-1}=\left[\begin{array}{cccccc}
* & * & \cdots & * & * & a_{n-1} \\
0 & * & \cdots & * & * & a_{n-2} \\
\vdots & \vdots & \ddots & \vdots & \vdots & \vdots \\
0 & 0 & \cdots & * & * & a_{2} \\
0 & 0 & \cdots & 0 & * & a_{1} \\
0 & 0 & \cdots & 0 & 0 & a_{0}
\end{array}\right]
$$

the last column entries of the inverse of $U$, i.e. the coefficients $a_{\ell}, \ell=0,1,2, \ldots, n-1$, may be calculated as follows

Lemma Appendix B.1. Let $U$ be defined as in (B.1), then the last column entries of $U^{-1}$ are

$$
\begin{aligned}
a_{0} & =\frac{1}{u_{n, n}} \\
a_{k} & =\sum_{j=0}^{k-1}-a_{j} \frac{u_{n-k, n-j}}{u_{n-k, n-k}} \\
k & =1,2, \ldots n
\end{aligned}
$$

the coefficients $a_{k}$ are defined as in (B.2).

Proof. It is known that the inverse of any non-singular matrix $R$ is $R^{-1}=\frac{1}{\operatorname{det}(R)} a d j(R)$, the non-singularity of $U$ guarantees that $\operatorname{det}(U) \neq 0$ moreover $\operatorname{det}(U)=\prod_{i=1}^{n} u_{i, i}$. By direct calculation, the $n, n$ entry of $U^{-1}$ is

$$
\begin{aligned}
a_{0} \triangleq & \frac{1}{\operatorname{det}(U)} \operatorname{adj}(U)_{n, n}=\frac{1}{u_{n, n}} \\
a_{1} \triangleq & \frac{1}{\operatorname{det}(U)} \operatorname{adj}(U)_{n-1, n}=\frac{u_{n-1, n}}{u_{n, n} u_{n-1, n-1}}=-a_{0} \frac{u_{n-1, n}}{u_{n-1, n-1}} \\
a_{2} \triangleq & \frac{1}{\operatorname{det}(U)} \operatorname{adj}(U)_{n-2, n}=\frac{u_{n-2, n-1} u_{n-1, n}-u_{n-2, n} u_{n-1, n-1}}{u_{n-2, n-2} u_{n-1, n-1} u_{n, n}}=-a_{1} \frac{u_{n-2, n-1}}{u_{n-2, n-2}}-a_{0} \frac{u_{n-2, n}}{u_{n-2, n-2}} \\
& \vdots \\
a_{i} \triangleq & \frac{1}{\operatorname{det}(U)} \operatorname{adj}(U)_{n-i, n}=-a_{i-1} \frac{u_{n-i, n-i-1}}{u_{n-i, n-i}}-a_{i-2} \frac{u_{n-i, n-i-2}}{u_{n-i, n-i}}-\cdots-a_{1} \frac{u_{n-i, n-1}}{u_{n-i, n-i}}-a_{0} \frac{u_{n-i, n}}{u_{n-i, n-i}}
\end{aligned}
$$

and the lemma follows.

\section{Appendix C. Proof of the second part of the Theorem 5.2}

In order to obtain the coefficients $c_{n}$ of Theorem 5.2 we must remember that each coefficient $c_{n}, n=$ $0,1, \ldots 2^{k}-1$, is the $2^{k}-n$ entry of the last column of $\bar{\Gamma}_{P}(\tau)$ and doing almost the same as for coefficients 
$b_{n}$ we have

$$
\begin{aligned}
c_{0}= & \frac{2^{2 k+2}}{2^{2 k+2}+\tau^{2}\left(\alpha+\beta p_{2^{k}}\right)} \\
c_{1}= & -4 \tau^{2} \frac{\alpha+\frac{\beta}{2}\left(p_{2^{k}-1}+p_{2^{k}}\right)}{2^{2 k+2}+\tau^{2}\left(\alpha+\beta p_{2^{k}-1}\right)} c_{0} \\
c_{2}= & 4 \tau^{2} \frac{-c_{0}\left(2 \alpha+\beta\left(\frac{1}{2} p_{2^{k}-2}+p_{2^{k}-1}+\frac{p_{2 k}}{2}\right)\right)-c_{1}\left(\alpha+\frac{\beta}{2}\left(p_{2^{k}-2}+p_{2^{k}-1}\right)\right)}{2^{2 k+2}+\tau^{2}\left(\alpha+\beta p_{2^{k}-2}\right)} \\
& \vdots \\
c_{n}= & \frac{4 \tau^{2}}{2^{2 k+2}+\tau^{2}\left(\alpha+\beta p_{2^{k}-n}\right)}\left(-\sum_{i=0}^{n-1}\left(c_{i}\left((n-i) \alpha-\frac{1}{2}\left(p_{2^{k}-n}+p_{2^{k}-i}\right)+\sum_{j=i}^{n} p_{2^{k}-j}\right)\right)\right)
\end{aligned}
$$

if we define

$$
\mu_{h}=\alpha+\frac{\beta}{2}\left(p_{2^{k}-h}+p_{2^{k}-h+1}\right)
$$

then the coefficients $c_{n}$ become

$$
\begin{aligned}
& c_{0}=\frac{2^{2 k+2}}{2^{2 k+2}+\tau^{2}\left(\alpha+\beta p_{2^{k}}\right)} \\
& c_{n}=-\psi_{n} \sum_{i=0}^{n-1}\left(c_{i} \sum_{j=i+1}^{n} \mu_{j}\right)
\end{aligned}
$$

for $n=1,2, \ldots 2^{k}-1, \psi_{h}$ is defined as in (5.9).

\section{Appendix D.}

In this part two Lemmas are stated: the first one says that the recurrent summations (D.1) can be written in terms of powers of the real constant $\delta$; and the second one gives us a rewriting of the multiple integrals shown in (D.2). The proof of the former is done by following the formulas (D.1), and the proof of the latter is done by rearranging the limits and integration variables. Both Lemmas are used in the proof of Theorem 5.4

Lemma Appendix D.1. Let

$$
\begin{aligned}
& A_{0}=B_{0}=1 \\
& A_{n}=1-\delta \sum_{i=0}^{n-1}\left[A_{i} \mathbb{I}_{n+1, i+1}\right] \\
& B_{n}=1-\delta\left[\sum_{i=0}^{n-1}(n-i) B_{i} \mathbb{I}_{i+2, i+1}\right]
\end{aligned}
$$

where $\mathbb{I}_{j, \ell}$ and $\delta$ are real constants, then $A_{n}$ and $B_{n}$ can be rewritten in terms of powers of $\delta$ as

$$
\begin{gathered}
A_{n}=1-\delta \sum_{i=1}^{n} \mathbb{I}_{n+1, i}+\delta^{2} \sum_{i=2}^{n} \mathbb{I}_{n+1, i} \sum_{j=1}^{i-1} \mathbb{I}_{i, j}-\delta^{3} \sum_{i=3}^{n} \mathbb{I}_{n+1, i} \sum_{j=1}^{i-1} \mathbb{I}_{i, j} \sum_{l=1}^{j} \mathbb{I}_{j, l}+\ldots \\
\ldots+(-1)^{\bar{n}} \delta^{\bar{n}} \sum_{i=\bar{n}}^{n} \mathbb{I}_{n+1, i} \sum_{j=1}^{i-1} \mathbb{I}_{i, j} \sum_{l=1}^{j} \mathbb{I}_{j, l} \ldots \sum_{y=1}^{x} \mathbb{I}_{x, y} \\
B_{n}=1-\delta \sum_{i=2}^{n+1} \mathbb{I}_{i, 1}+\delta^{2} \sum_{i=2}^{n} \mathbb{I}_{i, 1} \sum_{j=i+1}^{n+1} \mathbb{I}_{j, i}-\delta^{3} \sum_{i=2}^{n-1} \mathbb{I}_{i, 1} \sum_{j=i+1}^{n} \mathbb{I}_{j, i} \sum_{l=j+1}^{n+1} \mathbb{I}_{l, j}+\ldots \\
\ldots+(-1)^{\bar{n}} \delta^{\bar{n}} \sum_{i=2}^{n+2-\bar{n}} \mathbb{I}_{i, 1} \sum_{j=i+1}^{n+3-\bar{n}} \mathbb{I}_{j, i} \ldots \sum_{y=x+1}^{n+1} \mathbb{I}_{y, x}
\end{gathered}
$$


Proof. Following the formula for $S_{n}$ and grouping terms of powers of $\delta$ one obtains

$$
\begin{aligned}
A_{1}= & 1-\delta \mathbb{I}_{2,1} \\
A_{2}= & 1-\delta\left(\mathbb{I}_{3,1}+A_{1} \mathbb{I}_{3,2}\right)=1-\delta\left(\mathbb{I}_{3,1}+\mathbb{I}_{3,2}\right)+\delta^{2} \mathbb{I}_{2,1} \mathbb{I}_{3,2} \\
A_{3}= & 1-\delta\left(\mathbb{I}_{4,1}+\mathbb{I}_{4,2}+\mathbb{I}_{4,3}\right)+\delta^{2}\left(\mathbb{I}_{2,1} \mathbb{I}_{4,2}+\left(\mathbb{I}_{3,1}+\mathbb{I}_{3,2}\right) \mathbb{I}_{4,3}\right)-\delta^{3} \mathbb{I}_{2,1} \mathbb{I}_{3,2} \mathbb{I}_{4,3} \\
A_{4}= & 1-\delta\left(\mathbb{I}_{5,1}+A_{1} \mathbb{I}_{5,2}+A_{2} \mathbb{I}_{5,3}+A_{3} \mathbb{I}_{5,4}\right) \\
= & 1-\delta\left(\mathbb{I}_{5,1}+\mathbb{I}_{5,2}+\mathbb{I}_{5,3}+\mathbb{I}_{5,4}\right)+\delta^{2}\left(\mathbb{I}_{2,1} \mathbb{I}_{5,2}+\left(\mathbb{I}_{3,1}+\mathbb{I}_{3,2}\right) \mathbb{I}_{5,3}+\left(\mathbb{I}_{4,1}+\mathbb{I}_{4,2}+\mathbb{I}_{4,3}\right) \mathbb{I}_{5,4}\right)- \\
& -\delta^{3}\left(\mathbb{I}_{2,1} \mathbb{I}_{3,2} \mathbb{I}_{5,3}+\left(\mathbb{I}_{2,1} \mathbb{I}_{4,2}+\left(\mathbb{I}_{3,1}+\mathbb{I}_{3,2}\right) \mathbb{I}_{4,3}\right) \mathbb{I}_{5,4}\right)+\delta^{4} \mathbb{I}_{2,1} \mathbb{I}_{3,2} \mathbb{I}_{4,3} \mathbb{I}_{5,4} \\
& \vdots \\
A_{n}= & 1-\delta \sum_{i=1}^{n} \mathbb{I}_{n+1, i}+\delta^{2} \sum_{i=2}^{n} \mathbb{I}_{n+1, i} \sum_{j=1}^{i-1} \mathbb{I}_{i, j}-\delta^{3} \sum_{i=3}^{n} \mathbb{I}_{n+1, i} \sum_{j=1}^{i-1} \mathbb{I}_{i, j} \sum_{l=1}^{j} \mathbb{I}_{j, l}+\ldots+(-1)^{\bar{n}} \delta^{\bar{n}} \sum_{i=\bar{n}}^{n} \mathbb{I}_{n+1, i} \sum_{j=1}^{i-1} \mathbb{I}_{i, j} \sum_{l=1}^{j} \mathbb{I}_{j, l} \ldots \sum_{y=1}^{x} \mathbb{I}_{x, y}
\end{aligned}
$$

doing a similar procedure for $B_{n}$ one gets

$$
\begin{aligned}
& B_{0}= 1 \\
& B_{1}= 1-\delta \mathbb{I}_{2,1} \\
& B_{2}= 1-\delta\left(\mathbb{I}_{3,1}+\mathbb{I}_{2,1}\right)+\delta^{2} \mathbb{I}_{2,1} \mathbb{I}_{3,2} \\
& B_{3}= 1-\delta\left(\mathbb{I}_{4,1}+\mathbb{I}_{3,1}+\mathbb{I}_{2,1}\right)+\delta^{2}\left(\mathbb{I}_{2,1}\left(\mathbb{I}_{4,2}+\mathbb{I}_{3,2}\right)+\mathbb{I}_{3,1} \mathbb{I}_{4,3}\right)-\delta^{3} \mathbb{I}_{2,1} \mathbb{I}_{3,2} \mathbb{I}_{4,3} \\
& \vdots \\
& B_{n}= 1-\delta \sum_{i=2}^{n+1} \mathbb{I}_{i, 1}+\delta^{2} \sum_{i=2}^{n} \mathbb{I}_{i, 1} \sum_{j=i+1}^{n+1} \mathbb{I}_{j, i}-\delta^{3} \sum_{i=2}^{n-1} \mathbb{I}_{i, 1} \sum_{j=i+1}^{n} \mathbb{I}_{j, i} \sum_{l=j+1}^{n+1} \mathbb{I}_{l, j}+\ldots \\
& \ldots+(-1)^{\bar{n}} \delta^{\bar{n}} \sum_{i=2}^{n+2-\bar{n}} \mathbb{I}_{i, 1} \sum_{j=i+1}^{n+3-\bar{n}} \mathbb{I}_{j, i} \ldots \sum_{y=x+1}^{n+1} \mathbb{I}_{y, x}
\end{aligned}
$$

and the lemma follows.

Lemma Appendix D.2. Let the multiple definite integral sequence

$$
\begin{aligned}
A_{1}= & \int_{0}^{\tau} \int_{0}^{t_{1}} q\left(t_{2}\right) d t_{2} d t_{1}+\int_{0}^{\tau} \int_{t_{1}}^{\tau} q\left(t_{2}\right) d t_{2} d t_{1} \\
A_{2}= & \int_{0}^{\tau} \int_{0}^{t_{1}} \int_{t_{1}}^{\tau} \int_{t_{1}}^{t_{3}} q\left(t_{2}\right) q\left(t_{4}\right) d t_{4} d t_{3} d t_{2} d t_{1}+\int_{0}^{\tau} \int_{t_{1}}^{\tau} \int_{0}^{t_{1}} \int_{t_{3}}^{t_{1}} q\left(t_{2}\right) q\left(t_{4}\right) d t_{4} d t_{3} d t_{2} d t_{1} \\
A_{3}= & \int_{0}^{\tau} \int_{0}^{t_{1}} \int_{t_{1}}^{\tau} \int_{t_{1}}^{t_{3}} \int_{t_{3}}^{\tau} \int_{t_{3}}^{t_{5}} q\left(t_{2}\right) q\left(t_{4}\right) q\left(t_{6}\right) d t_{6} d t_{5} d t_{4} d t_{3} d t_{2} d t_{1}+ \\
& \quad+\int_{0}^{\tau} \int_{t_{1}}^{\tau} \int_{0}^{t_{1}} \int_{t_{3}}^{t_{1}} \int_{0}^{t_{3}} \int_{t_{5}}^{t_{3}} q\left(t_{2}\right) q\left(t_{4}\right) q\left(t_{6}\right) d t_{6} d t_{5} d t_{4} d t_{3} d t_{2} d t_{1}
\end{aligned}
$$

and the general term

$$
\begin{aligned}
A_{\bar{n}}= & \int_{0}^{\tau} \int_{0}^{t_{1}} \int_{t_{1}}^{\tau} \int_{t_{1}}^{t_{3}} \ldots \int_{t_{2 \bar{n}-3}}^{\tau} \int_{t_{2 \bar{n}-3}}^{t_{2 \bar{n}-1}} q\left(t_{2}\right) \ldots q\left(t_{2 \bar{n}-2}\right) q\left(t_{2 \bar{n}}\right) d t_{2 \bar{n}} d t_{2 \bar{n}-1} \ldots d t_{4} d t_{3} d t_{2} d t_{1}+ \\
& +\int_{0}^{\tau} \int_{t_{1}}^{\tau} \int_{0}^{t_{1}} \int_{t_{3}}^{t_{2}} \ldots \int_{0}^{t_{2 \bar{n}-3}} \int_{t_{2 \bar{n}-1}}^{t_{2 \bar{n}-3}} q\left(t_{2}\right) \ldots q\left(t_{2 \bar{n}-2}\right) q\left(t_{2 \bar{n}}\right) d t_{2 \bar{n}} d t_{2 \bar{n}-1} \ldots d t_{4} d t_{3} d t_{2} d t_{1}
\end{aligned}
$$


for $\bar{n}=2,3,4$, then, the sequence may be written as

$$
\begin{aligned}
& A_{1}=\tau \int_{0}^{\tau} q\left(t_{2}\right) d t_{2} \\
& A_{2}=\int_{0}^{\tau} \int_{0}^{t_{2}}\left(\tau-t_{2}+t_{1}\right)\left(t_{2}-t_{1}\right) p\left(t_{1}\right) p\left(t_{2}\right) d t_{1} d t_{2} \\
& A_{3}=\int_{0}^{\tau} \int_{0}^{t_{6}} \int_{0}^{t_{4}}\left(\tau-t_{6}+t_{2}\right)\left(t_{6}-t_{4}\right)\left(t_{4}-t_{2}\right) q\left(t_{2}\right) q\left(t_{4}\right) q\left(t_{6}\right) d t_{2} d t_{4} d t_{6}
\end{aligned}
$$

and the general term

$$
A_{n}=\int_{0}^{\tau} d t_{1} \int_{0}^{t_{1}} d t_{2} \ldots \int_{0}^{t_{\bar{n}-1}}\left(\tau-t_{1}+t_{n}\right)\left(t_{1}-t_{2}\right) \ldots\left(t_{n-1}-t_{n}\right) q\left(t_{1}\right) q\left(t_{2}\right) \ldots q\left(t_{n}\right) d t_{n}
$$

Proof. By rearranging the integration variables of the three first coefficients given in (D.2) we get

$$
\begin{gathered}
A_{1}=\int_{0}^{\tau} \int_{0}^{t_{1}} q\left(t_{2}\right) d t_{2} d t_{1}+\int_{0}^{\tau} \int_{t_{1}}^{\tau} q\left(t_{2}\right) d t_{2} d t_{1} \\
=\int_{0}^{\tau} \int_{0}^{\tau} q\left(t_{2}\right) d t_{2} d t_{1} \\
=\int_{0}^{\tau} \int_{0}^{\tau} q\left(t_{2}\right) d t_{1} d t_{2} \\
=\tau \int_{0}^{\tau} q\left(t_{2}\right) d t_{2} \\
A_{2}=\int_{0}^{\tau} \int_{0}^{t_{1}} \int_{t_{1}}^{\tau} \int_{t_{1}}^{t_{3}} q\left(t_{2}\right) q\left(t_{4}\right) d t_{4} d t_{3} d t_{2} d t_{1}+\int_{0}^{\tau} \int_{t_{1}}^{\tau} \int_{0}^{t_{1}} \int_{t_{3}}^{t_{1}} q\left(t_{2}\right) q\left(t_{4}\right) d t_{4} d t_{3} d t_{2} d t_{1} \\
=\int_{0}^{\tau} \int_{0}^{t_{4}}\left(\tau-t_{4}\right)\left(t_{4}-t_{2}\right) q\left(t_{2}\right) q\left(t_{4}\right) d t_{2} d t_{4}+\int_{\delta}^{\tau} \int_{0}^{t_{4}}\left(t_{4}-t_{2}\right) t_{2} q\left(t_{2}\right) q\left(t_{4}\right) d t_{2} d t_{4} \\
=\int_{0}^{\tau} \int_{0}^{t_{2}}\left(\tau-t_{2}+t_{1}\right)\left(t_{2}-t_{1}\right) q\left(t_{1}\right) q\left(t_{2}\right) d t_{1} d t_{2} \\
A_{3}=\int_{0}^{\tau} \int_{0}^{t_{1}} \int_{t_{1}}^{\tau} \int_{t_{1}}^{t_{3}} \int_{t_{3}}^{\tau} \int_{t_{3}}^{t_{5}} q\left(t_{2}\right) q\left(t_{4}\right) q\left(t_{6}\right) d t_{6} d t_{5} d t_{4} d t_{3} d t_{2} d t_{1}+ \\
+\int_{0}^{\tau} \int_{t_{1}}^{\tau} \int_{0}^{t_{1}} \int_{t_{3}}^{t_{1}} \int_{0}^{t_{3}} \int_{t_{5}}^{t_{3}} q\left(t_{2}\right) q\left(t_{4}\right) q\left(t_{6}\right) d t_{6} d t_{5} d t_{4} d t_{3} d t_{2} d t_{1} \\
=\int_{0}^{\tau} \int_{0}^{t_{6}} \int_{0}^{t_{4}}\left(\tau-t_{6}\right)\left(t_{6}-t_{4}\right)\left(t_{4}-t_{2}\right) q\left(t_{2}\right) q\left(t_{4}\right) q\left(t_{6}\right) d t_{2} d t_{4} d t_{6}+ \\
+\int_{0}^{\tau} \int_{0}^{t_{2}} \int_{0}^{t_{4}}\left(t_{4}-t_{6}\right)\left(t_{2}-t_{4}\right) t_{6} q\left(t_{2}\right) q\left(t_{4}\right) q\left(t_{6}\right) d t_{6} d t_{4} d t_{2} \\
\int_{6}^{t_{6}} \int_{0}^{t_{4}}\left(\tau-t_{6}+t_{2}\right)\left(t_{6}-t_{4}\right)\left(t_{4}-t_{2}\right) q\left(t_{2}\right) q\left(t_{4}\right) q\left(t_{6}\right) d t_{2} d t_{4} d t_{6}
\end{gathered}
$$

and so on. The general term is then

$$
A_{n}=\int_{0}^{\tau} d t_{1} \int_{0}^{t_{1}} d t_{2} \ldots \int_{0}^{t_{\bar{n}-1}}\left(\tau-t_{1}+t_{n}\right)\left(t_{1}-t_{2}\right) \ldots\left(t_{n-1}-t_{n}\right) q\left(t_{1}\right) q\left(t_{2}\right) \ldots q\left(t_{n}\right) d t_{n}
$$

and the lemma follows. 


\section{References}

\section{References}

[1] J. A. Richards, Analysis of periodically time-varying systems, Springer Science \& Business Media, 2012.

[2] R. Dvorak, C. Lhotka, Celestial dynamics: chaoticity and dynamics of celestial systems, John Wiley \& Sons, 2013.

[3] A. M. Lyapunov, The general problem of the stability of motion, International Journal of Control 55 (3).

[4] D. G. Luenberger, Optimization by vector space methods, John Wiley \& Sons, 1969.

[5] A. M. Lyapunov, On the series, encountered in the theory of linear second order differential equations with periodic coefficients (in russian), Academy of Science notes on the physics-mathematical department VIII (1902) 1-70.

[6] H. Hochstadt, Asymptotic estimates for the Sturm-Liouville spectrum, Communications on Pure and Applied Mathematics 14 (4) (1961) 749-764.

[7] J. Shi, A new form of discriminant for Hill equation, Ann. of Diff. Eqs 15 (2) (1999) 191-210.

[8] W. Magnus, S. Winkler, Hill's equation, Dover Publications, 2013.

[9] I. Gel'fand, V. Lidskii, On the structure of the regions of stability of linear canonical systems of differential equations with periodic coefficients, Amer. Math. Soc. Transl. 8 143-181.

[10] V. Yakubovich, V. Starzhinskii, Linear differential equations with periodic coefficients 2 vols., Wiley (New York), 1975.

[11] R. Brockett, Finite dimensional linear systems, Wiley, 1970.

[12] K. Meyer, G. Hall, D. Offin, Introduction to Hamiltonian dynamical systems and the N-body problem, Vol. 90, Springer Science \& Business Media, 2008.

[13] P. Lancaster, M. Tismenetsky, The theory of matrices: with applications, Elsevier, 1985.

[14] H. Hochstadt, Functiontheoretic properties of the discriminant of Hill's equation, Mathematische Zeitschrift 82 (3) (1963) $237-242$

[15] K. G. Beauchamp, Walsh functions and their applications, Vol. 3, Academic press, 1975.

[16] H. F. Harmuth, Sequency theory: foundations and applications, Advances in Electronics and Electron Physics Suppl., New York: Academic Press, 1977.

[17] N. J. Fine, On the Walsh functions, Transactions of the American Mathematical Society 65 (3) (1949) $372-414$.

[18] P. J. Collins, Differential and integral equations, Oxford University Press, 2006.

[19] L. N. Trefethen, M. Embree, Spectra and pseudospectra: the behavior of nonnormal matrices and operators, Princeton University Press, 2005.

[20] J.-H. He, Homotopy perturbation technique, Computer methods in applied mechanics and engineering 178 (3) (1999) $257-262$.

[21] V. Karanam, P. Frick, R. Mohler, Bilinear system identification by Walsh functions, IEEE Transactions on Automatic Control 23 (4) (1978) 709-713.

[22] M. Gulamhusein, Simple matrix-theory proof of the discrete dyadic convolution theorem, Electronics Letters 9 (10) (1973) $238-239$.

[23] C. Cheng, Y. Tsay, T. Wu, Walsh operational matrices for fractional calculus and their application to distributed systems, Journal of the Franklin Institute 303 (3) (1977) 267-284.

[24] P. Stavroulakis, S. Tzafestas, Distributed-parameter observer-based control implementation using finite spatial measurements, Mathematics and Computers in Simulation 22 (4) (1980) 373-379. 